\title{
Regio- and Diastereoselective Decarboxylative Coupling of Heteroaromatic Alkanes
}

Shelli R. Waetzig and Jon A. Tunge*

Department of Chemistry, University of Kansas, Lawrence, KS 66045

\section{Supporting Information}

\section{Table of Contents}

General Experimental

S-1

Table 1: Impact of catalyst loading on elimination $\quad$ S-3

Spectral Characterization of 2a $\quad$ S-4

Spectral Characterization of $\mathbf{2 b} \quad$ S-5

Spectral Characterization of 2c $\quad$ S-6

$\begin{array}{ll}\text { Spectral Characterization of 2d } & \text { S-7 }\end{array}$

Spectral Characterization of $2 \mathbf{e} \quad$ S-8

Spectral Characterization of $\mathbf{2 f} \quad$ S-9

Spectral Characterization of $\mathbf{2 g} \quad$ S-10

Spectral Characterization of $\mathbf{2 h} \quad$ S-11

Spectral Characterization of $2 \mathbf{i} \quad$ S-12

Spectral Characterization of $\mathbf{2 j} \quad$ S-13

Spectral Characterization of $\mathbf{2 k} \quad$ S-14

Spectral Characterization of 2l $\quad$ S-15

$\begin{array}{ll}\text { Spectral Characterization of } \mathbf{2 m} & \text { S-16 }\end{array}$

$\begin{array}{ll}\text { Spectral Characterization of } \mathbf{2 n} & \text { S-17 }\end{array}$

$\begin{array}{ll}\text { Spectral Characterization of } 20 & \text { S-18 }\end{array}$

$\begin{array}{ll}\text { Spectral Characterization of } \mathbf{2 p} & \text { S-19 }\end{array}$

$\begin{array}{ll}\text { Spectral Characterization of } \mathbf{2 q} & \text { S-20 }\end{array}$

$\begin{array}{ll}\mathrm{X} \text {-ray crystal structure for } \mathbf{4 g} & \mathrm{S}-21\end{array}$

\section{Experimental:}

\section{General Information:}

All reactions were run in flame-dried glassware under Ar atmosphere using standard Schlenk techniques. $\mathrm{CH}_{2} \mathrm{Cl}_{2}$, Toluene and THF were dried over activated alumina. Commercially available reagents were used without additional purification unless otherwise stated. Compound purification was effected by flash chromatography using 230x400 mesh, 60 Å porosity, silica obtained from Sorbent Technologies. ${ }^{1} \mathrm{H}$ NMR and ${ }^{13} \mathrm{C}$ NMR spectra were obtained on a Bruker Avance 400 or a Bruker Avance 500 DRX spectrometer and referenced to residual protio solvent signals (some spectra were taken using a QNP Cryoprobe). Structural assignments are based on ${ }^{1} \mathrm{H},{ }^{13} \mathrm{C}$, DEPT-135, COSY, HSQC and IR spectroscopies. 
Procedure for the synthesis of allyl-2-benzoxazole propanoates: This was prepared using a modification of a procedure by Cherney. ${ }^{1}$ Dimethyl malonate $(13.8 \mathrm{mmol})$ was added to 2-aminophenol $(4.6 \mathrm{mmol})$. The mixture was heated to $160^{\circ} \mathrm{C}$ for 5 hours, at which time $\mathrm{p}$ - $\mathrm{TsOH}(0.46 \mathrm{mmol})$ was added to the reaction. This solution was stirred at $160^{\circ} \mathrm{C}$ for 12 hours. The mixture was cooled to room temperature and directly purified on silica gel using 95:5 hexane/ethyl acetate as the eluent. The resulting methyl-2benzoxazol-2-yl acetate was then treated with $\mathrm{LiOH}$ according to the procedure by Cherney and let stir at room temperature overnight. ${ }^{1}$ The crude acid was treated with 1.1 equivalents of the appropriate allylic alcohol, 1.0 equivalents of DCC, and 0.10 equivalents of DMAP in $\mathrm{CH}_{2} \mathrm{Cl}_{2}{ }^{2}$. The solutions were stirred at room temperature overnight. The reactions were filtered and washed with $\mathrm{H}_{2} \mathrm{O}$ and brine, dried over $\mathrm{MgSO}_{4}$, and concentrated. The allyl-2-benzoxazol-2-yl acetates were purified on silica gel using 90:10 hexane/ethyl acetate. Alkylation of the $\alpha$-position was accomplished using ${ }^{\mathrm{t}} \mathrm{BuOK}$ and the corresponding alkylating agent. ${ }^{3}$

Procedure for the synthesis of allyl-2-benzothiazole propanoates: A mixture of the appropriate allyl cyanoacetate ${ }^{4}$ was mixed with 2 -aminothiophenol ${ }^{5}$ and heated at $120^{\circ} \mathrm{C}$ overnight. ${ }^{6}$ The crude reaction was purified directly on silica gel. The resulting ester was alkylated with ${ }^{\mathrm{t}} \mathrm{BuOK}$ and the appropriate alkylating agent. ${ }^{3}$

Procedure for the synthesis of allyl-2-benzimidazole propanoates: Benzimidazolyl acetonitrile was hydrolyzed to the corresponding acid according to Copeland and Day. ${ }^{7}$ Coupling of the acid was achieved using cinnamyl alchohol, DCC, $\mathrm{Et}_{3} \mathrm{~N}$ and cat. DMAP after heating at reflux in $\mathrm{CH}_{2} \mathrm{Cl}_{2}$ overnight. ${ }^{2}$ The benzimidazole nitrogen was then alkylated with MeI according to Das and coworkers. ${ }^{8}$ Alkylation was achieved with ${ }^{\mathrm{t}} \mathrm{BuOK}$ and the appropriate alkylating agent. ${ }^{3}$

Procedure for the synthesis of allyl-2-pyridin-2-yl propanoates: These substrates were synthesized by the DCC/DMAP catalyzed coupling of 2-pyridyl acetic acid hydrochloride with the appropriate allylic alcohol. ${ }^{2}$ Alkylation was achieved with ${ }^{t} \mathrm{BuOK}$ and the appropriate alkylating reagent. ${ }^{3}$

Procedure for the synthesis of cinnamyl 2-(4,5-diphenyloxazol-2-yl -3phenylpropanoate: The methyl 2(4,5-diphenyloxazol-2-yl)acetate was synthesized in a procedure described by Davies. ${ }^{9}$ The ester was hydrolyzed using LiOH. ${ }^{1}$ The crude acid

\footnotetext{
${ }^{1}$ Cherney, R. J. PCT Intl. App. \# WO 2004098516, 2004.

${ }^{2}$ Kim, H. Y.; Lantrip, D. A.; Fuchs, P. L. Org. Lett. 2001, 3, 2137.

${ }^{3}$ Lee, H.-S.; Park, J.-S.; Kim, B. M.; Gellman, S. H. J. Org. Chem. 2003, 68, 1575.

${ }^{4}$ Kobayashi, T.; Inoue, T.; Nishino, S.; Fujihara, Y.; Oizumi, K.; Kimura, T. Chem. Pharm. Bull. $1995,43$. 797.

5 2-aminothiophenol was purified via distillation and stored under Ar atmosphere.

${ }^{6}$ Abbotto, A.; Bradamante, S.; Facchetti, A.; Pagani, G. A. J. Org. Chem. 2002, 67, 5753.

${ }^{7}$ Copeland, R. A. B.; Day, A. R. J. Amer. Chem. Soc. 1943, 65, 1072.

${ }^{8}$ Das, J.; Laxman Rao, C. V.; Sastry, T. V. R. S.; Roshaiah, M.; Gowri Sankar, P.; Khadeer, A.; Sitaram Kumar, M.; Mallik, A.; Selvakumar, N.; Iqbal, J.; Trehan, S. Bioorg. Med. Chem. Lett. 2005, $15,337$.

${ }^{9}$ Davies, H. M.; Townsend, R. J. J. Org. Chem. 2001, 66, 6595.
} 
was coupled with cinnamyl alcohol using DCC/DMAP catalyzed reaction. ${ }^{2}$ Alkylation was achieved using ${ }^{\mathrm{t}} \mathrm{BuOK}$ and benzyl bromide. ${ }^{3}$

Procedure for the bromocyclization of $2 \mathrm{~g}: \mathbf{2 g}\left(0.06 \mathrm{M}\right.$ in $\left.\mathrm{C}_{6} \mathrm{H}_{6}\right)$ was treated with bromine ( 1 equiv., $0.07 \mathrm{M}$ solution in $\mathrm{CH}_{2} \mathrm{Cl}_{2}$ ) in at $0^{\circ} \mathrm{C}$. The reaction was then stirred at room temperature for $15 \mathrm{~min}$ and then filtered. The filtrate was recrystallized from $\mathrm{CH}_{2} \mathrm{Cl}_{2}$ /pentane. ${ }^{10}$

General Procedure for Palladium-catalyzed decarboxylation reaction: In a dried Schlenk tube under argon, $\mathrm{Pd}\left(\mathrm{PPh}_{3}\right)_{3}(0.013 \mathrm{mmol})$ was added to substrates $1(0.25$ mmol) in toluene. The reactions of the cinnamyl esters were run at $0.05 \mathrm{M}$ substrate concentration and $80^{\circ} \mathrm{C}$ for the specified time. The reactions of the alkyl substituted allyl esters were run at $0.025 \mathrm{M}$ substrate concentration at $100^{\circ} \mathrm{C}$ for the specified time. After such time, the reactions were cooled to room temperature, concentrated in vacuo and directly purified via flash chromatography on silica gel.

\section{Table of Effect of Catalyst Loading on Elimination:}

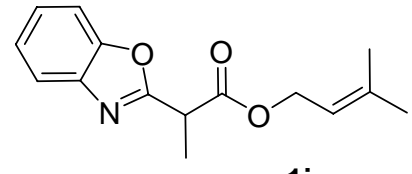

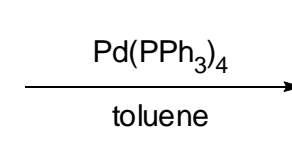

\begin{tabular}{cc}
{$[\mathbf{1 j}]$} & $\mathrm{mol} \% \mathrm{c}$ \\
\hline 0.05 & $0.5^{\mathrm{a}}$ \\
0.05 & 1 \\
0.05 & 5 \\
0.05 & 10
\end{tabular}

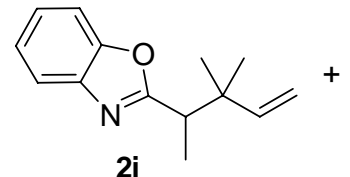

2j

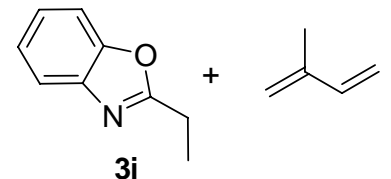

3j

a Reaction only went to $42 \%$ completion after $16 \mathrm{~h}$.

${ }^{10}$ Szychowski, J.; Wrobel, J. T.; Leniewski, A. Bull. Acad. Pol. Sci., Ser. Sci. Chim. 1980, 28, 9. 


\section{Spectral Characterization of Compounds:}

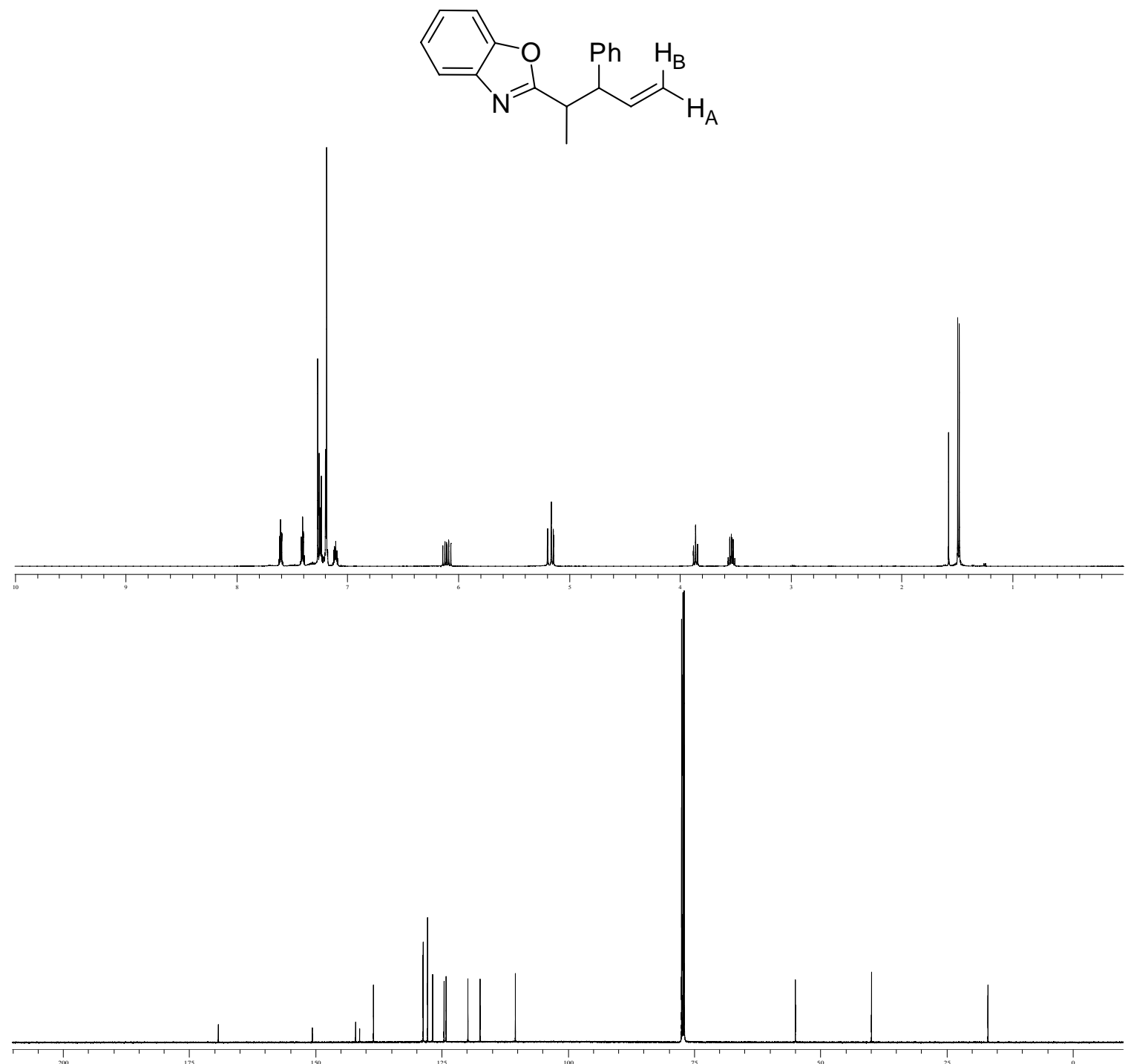

2a: (2-(3-phenylpent-4-en-2-yl)benzoxaole): white solid: 98:2 hex/EtOAc: ${ }^{1} \mathrm{H}$ NMR $\left(500 \mathrm{MHz}, \mathrm{CDCl}_{3}\right) \delta 1.49$ (d, 3H: $\left.J=7.0 \mathrm{~Hz}, \mathrm{R}_{2} \mathrm{CHCH}_{3}\right), 3.53$ (dq, $1 \mathrm{H}: J=7.0,9.1 \mathrm{~Hz}$, $\left.\mathrm{R}_{2} \mathrm{CHCH}_{3}\right), 3.86\left(\mathrm{t}, 1 \mathrm{H}: J=9.1 \mathrm{~Hz}, \mathrm{R}_{2} \mathrm{CHPh}\right), 5.15\left(\mathrm{dd}, 1 \mathrm{H}: J=1.5,10.1 \mathrm{~Hz}, \mathrm{H}_{\mathrm{A}}\right), 5.19$ (dd, $1 \mathrm{H}: J=1.5,17.0 \mathrm{~Hz}, \mathrm{H}_{\mathrm{B}}$ ), 6.10 (ddd, $1 \mathrm{H}: J=9.1,10.0,17.0 \mathrm{~Hz}, \mathrm{RCH}=\mathrm{CH}_{2}$ ), 7.11 (m, 1H: $\mathrm{Ar} \mathrm{CH}$ ), 7.13 (s, 2H: overlapping Ar CH's), 7.19 (d, 1H: J=1.5 Hz, Ar CH), 7.24 (m, 2H: overlapping Het Ar CH's), 7.41 (m, 1H: Het Ar CH's), 7.60 (m, 1H: Het Ar CH); ${ }^{13} \mathrm{C}$ NMR $\left(125 \mathrm{MHz}, \mathrm{CDCl}_{3}\right) \delta 16.9\left(\mathrm{R}_{2} \mathrm{CHCH}_{3}\right), 40.0\left(\mathrm{R}_{2} \mathrm{CHCH}_{3}\right), 55.3\left(\mathrm{R}_{2} \mathrm{CHPh}\right)$, 111.1 (Het Ar CH), $118.1\left(\mathrm{RCH}=\mathrm{CH}_{2}\right), 120.5$ (Het Ar CH), 124.9 (Het Ar CH), 125.2 (Het $\mathrm{Ar} \mathrm{CH}), 127.5$ (Ar CH), $128.6(\mathrm{Ar} \mathrm{CH}), 129.4(\mathrm{Ar} \mathrm{CH}), 139.3\left(\mathrm{RCH}=\mathrm{CH}_{2}\right), 142.1$ (quat. $\mathrm{Ar} \mathrm{C}$ ), 142.9 (quat. $\mathrm{Ar} \mathrm{C}$ ), 151.5 (quat. $\mathrm{Ar} \mathrm{C}$ ), $170.3(\mathrm{~N}=\mathrm{CO}) ; \mathrm{IR}\left(\mathrm{CH}_{2} \mathrm{Cl}_{2}\right) v_{\max }$ : 3054, 2986, 1638, 1613, 1569, 1456, 1421, 1263, 1152, 994, 926, 896; HRMS calcd for $[\mathrm{M}+\mathrm{H}]$ 264.1388, found 264.1382. 


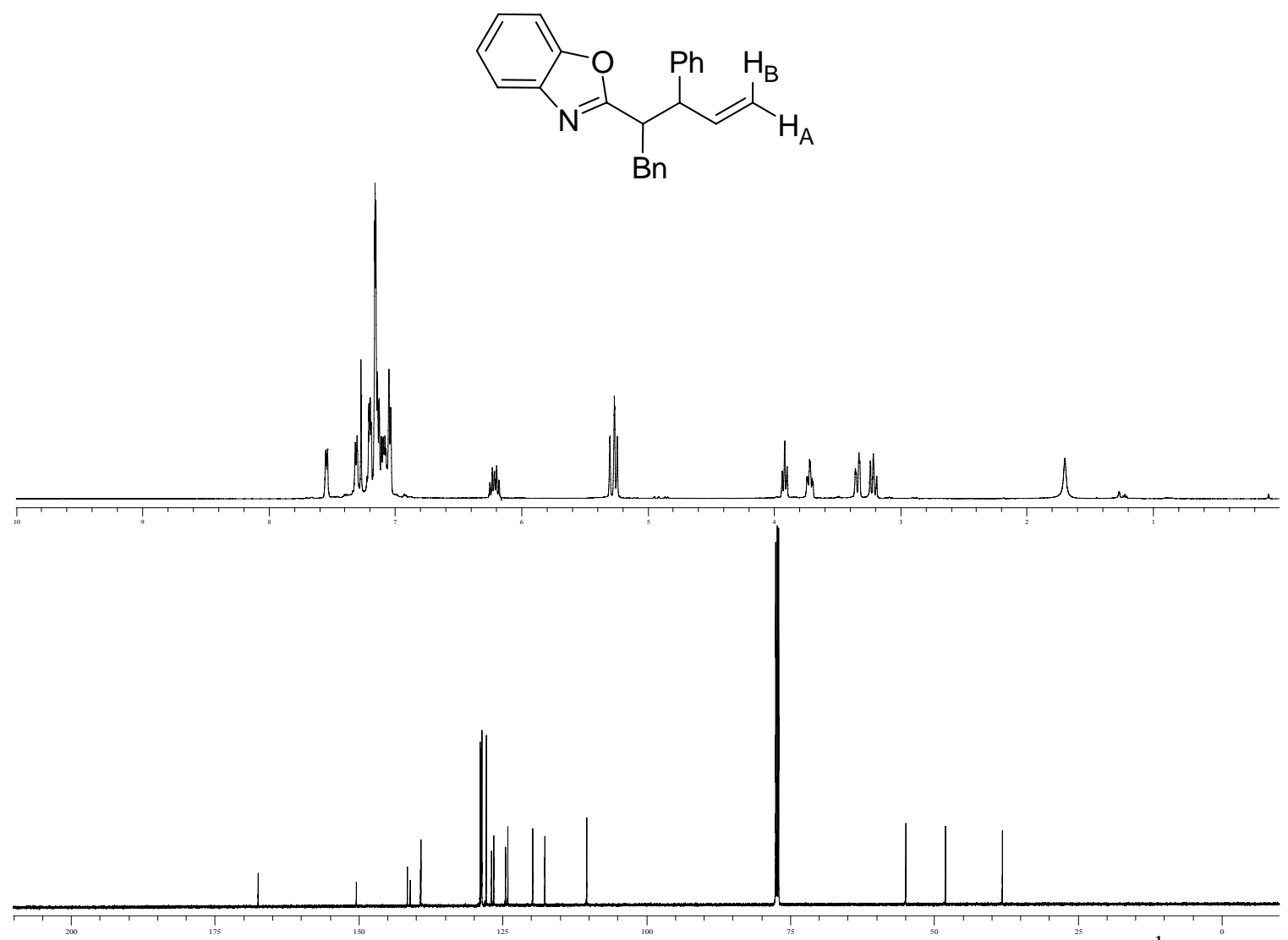

2b: (2-(1,3-diphenylpent-4-en-2-yl)benzoxazole): white solid: 98:2 hex/EtOAc: ${ }^{1} \mathrm{H}$ NMR $\left(500 \mathrm{MHz}, \mathrm{CDCl}_{3}\right) \delta 3.21$ (dd, $\left.1 \mathrm{H}: J=10.9,13.9 \mathrm{~Hz}, \mathrm{CHHPh}\right), 3.34$ (dd, $1 \mathrm{H}: J=4.0$, $13.9 \mathrm{~Hz}, \mathrm{CHHPh}$ ), 3.72 (ddd, $1 \mathrm{H}: J=4.0,9.6,10.9 \mathrm{~Hz}, \mathrm{R}_{2} \mathrm{CHCH}_{2} \mathrm{Ph}$ ), 3.92 (t, 1H: $J=$ $9.6 \mathrm{~Hz}, \mathrm{R}_{2} \mathrm{CHPh}$ ), 5.25 (app. d, $1 \mathrm{H}: J=10.0 \mathrm{~Hz}, \mathrm{H}_{\mathrm{A}}$ ), 5.29 (app. d, $1 \mathrm{H}: J=17.0 \mathrm{~Hz}, \mathrm{H}_{\mathrm{B}}$ ), 6.22 (ddd, $\left.1 \mathrm{H}: J=1.3,10.0,17.0 \mathrm{~Hz}, \mathrm{RCH}=\mathrm{CH}_{2}\right), 7.03-7.21(\mathrm{~m}, 12 \mathrm{H}$ : overlapping $\mathrm{Ar}$ CH's), 7.31 (m, 1H: Het Ar CH), 7.55 (m, 1H: Het Ar CH); ${ }^{13} \mathrm{C}$ NMR (125 MHz, CDCl 3 ) $\delta 38.3\left(\mathrm{PhCH}_{2} \mathrm{R}\right), 48.3\left(\mathrm{R}_{2} \mathrm{CHCH}_{2} \mathrm{Ph}\right), 55.2\left(\mathrm{R}_{2} \mathrm{CHPh}\right), 111.0(\mathrm{Het} \mathrm{Ar} \mathrm{CH}), 118.3$ $\left(\mathrm{RCH}=\mathrm{CH}_{2}\right), 120.5$ (Het Ar CH), 124.8 (Het Ar CH), 125.2 (Het Ar CH), 127.3 (Ar $\mathrm{CH}), 127.7$ (Ar CH), 128.6 (Ar CH), 129.4 (Ar CH), 129.5 (Ar CH), $129.7(\mathrm{Ar} \mathrm{CH})$, $140.0\left(\mathrm{RCH}=\mathrm{CH}_{2}\right), 140.1$ (quat. $\mathrm{Ar} \mathrm{C}$ ), 141.9 (quat. Ar C), 142.4 (quat. Ar C), 151.3 (quat. $\mathrm{Ar} \mathrm{C}), 168.5(\mathrm{OC}=\mathrm{N})$; IR $\left(\mathrm{CH}_{2} \mathrm{Cl}_{2}\right)$ v max $_{\max }$ 3054, 2985, 1637, 1612, 1570, 1495, $1455,1421,1263,1144,992,926,896$; HRMS calcd for $[\mathrm{M}+\mathrm{H}] 340.1701$, found 340.1712 . 


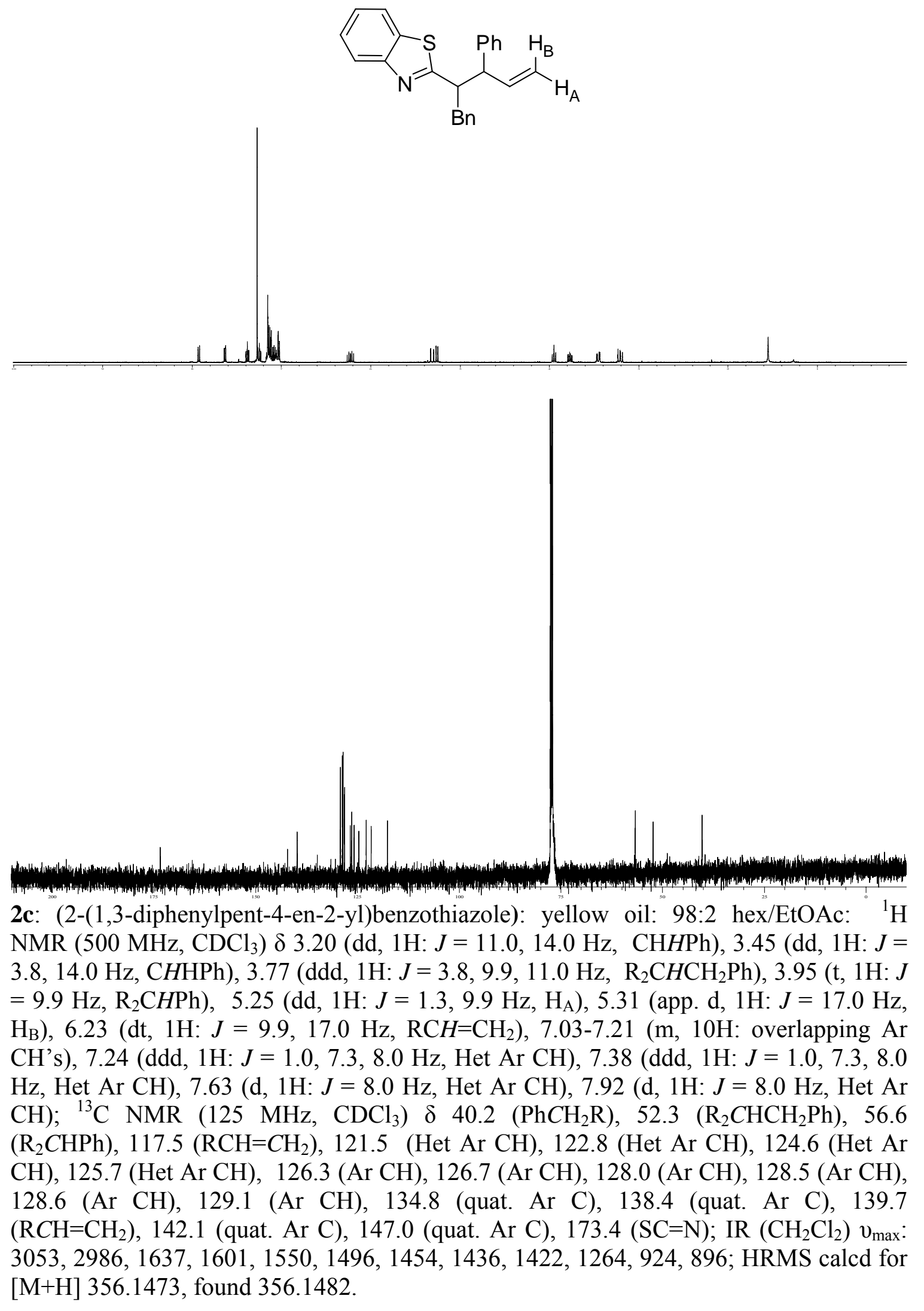




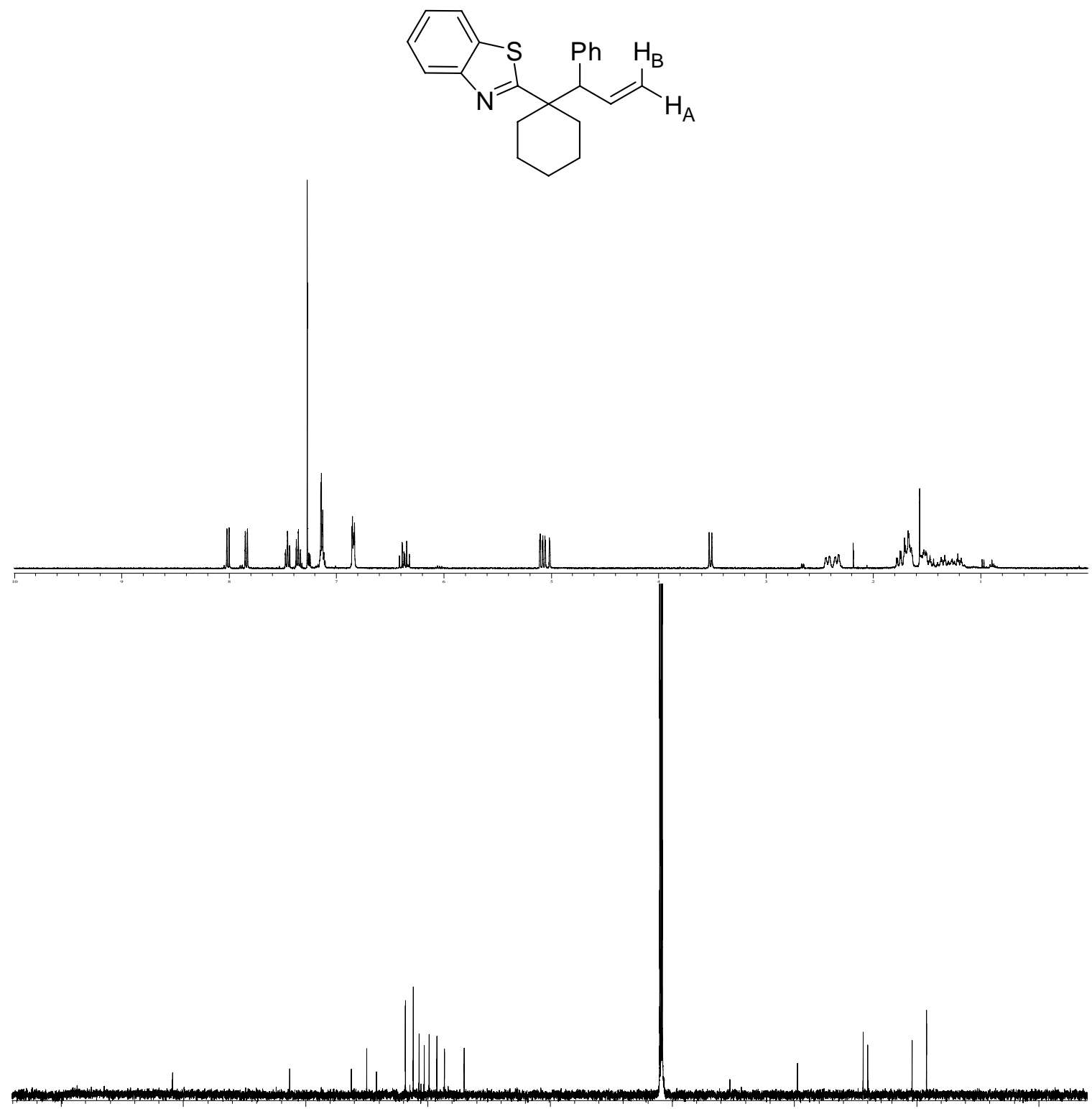

2d: (2-(1-(1-phenylallyl)cyclohexyl)benzothiazole): pale yellow oil: 98:2 hex/EtOAc: ${ }^{1} \mathrm{H}$ $\mathrm{NMR}\left(400 \mathrm{MHz}, \mathrm{CDCl}_{3}\right) \delta$ 1.15-1.40 (m, 2H: overlapping cyclohexyl CH's), 1.44-1.56 (m, 2H: overlapping cyclohexyl CH's), 1.63-1.79 (m, 4H: overlapping cyclohexyl CH's), 2.34 (app. d, 1H: $J=12.5 \mathrm{~Hz}$, cyclohexyl CH), 2.42 (app. d, 1H: $J=13.2 \mathrm{~Hz}$, cyclohexyl $\mathrm{CH}$ ), 3.52 (d, 1H: $J=9.9 \mathrm{~Hz}, \mathrm{R}_{2} \mathrm{CHPh}$ ), 5.03 (app. dd, 1H: $J=1.7,16.7 \mathrm{~Hz}, \mathrm{H}_{\mathrm{B}}$ ), 5.09 (dd, 1H: $J=1.7,10.1 \mathrm{~Hz}, \mathrm{H}_{\mathrm{A}}$ ), 6.36 (dt, 1H: $\left.J=10.1,16.7 \mathrm{~Hz}, \mathrm{RCH}=\mathrm{CH}_{2}\right) 6.84$ (dd, 2H: $J=1.8,7.7 \mathrm{~Hz}$, overlapping Ar CH's), 7.13 (m, 3H: overlapping Ar CH's), 7.35 (app. t, 1H: $J=7.8 \mathrm{~Hz}$, Het Ar CH), 7.45 (app. t, 1H: $J=7.8 \mathrm{~Hz}$, Het Ar CH) 7.84 (d, 1H: $J=7.8$ $\mathrm{Hz}$, Het Ar CH), 8.01 (d, $1 \mathrm{H}: J=7.8 \mathrm{~Hz}$, Het Ar CH); ${ }^{13} \mathrm{C} \mathrm{NMR}\left(125 \mathrm{MHz}, \mathrm{CDCl}_{3}\right) \delta$ 22.9 (overlapping cyclohexyl $\mathrm{CH}_{2}$ 's), 25.9 (cyclohexyl $\mathrm{CH}_{2}$ ), 24.9 (cyclohexyl $\mathrm{CH}_{2}$ ), 35.9 (cyclohexyl $\left.\mathrm{CH}_{2}\right), 49.3$ (quat. cyclohexyl C), $63.1\left(\mathrm{R}_{2} \mathrm{CHPh}\right), 117.5\left(\mathrm{RCH}=\mathrm{CH}_{2}\right)$, 121.6 (Het Ar CH), 123.1 (Het Ar CH), 124.7 (Het Ar CH), 125.7 (Het Ar CH), 126.7 $(\mathrm{Ar} \mathrm{CH}), 127.9(\mathrm{Ar} \mathrm{CH}), 129.5(\mathrm{Ar} \mathrm{CH}), 135.5(\mathrm{Ar} \mathrm{CH}), 137.4\left(\mathrm{RCH}=\mathrm{CH}_{2}\right), 140.6$ (quat. 
Ar C), 153.2 (quat. Ar C), $177.2(\mathrm{SC}=\mathrm{N})$; IR $\left(\mathrm{CH}_{2} \mathrm{Cl}_{2}\right)$ v max $: 3054,2986,2933,2857$, 1633, 1600, 1492, 1451, 1421, 1313, 1264, 1047, 1013, 919, 896; HRMS calcd for $[\mathrm{M}+\mathrm{H}] 343.1630$, found 343.1630 .

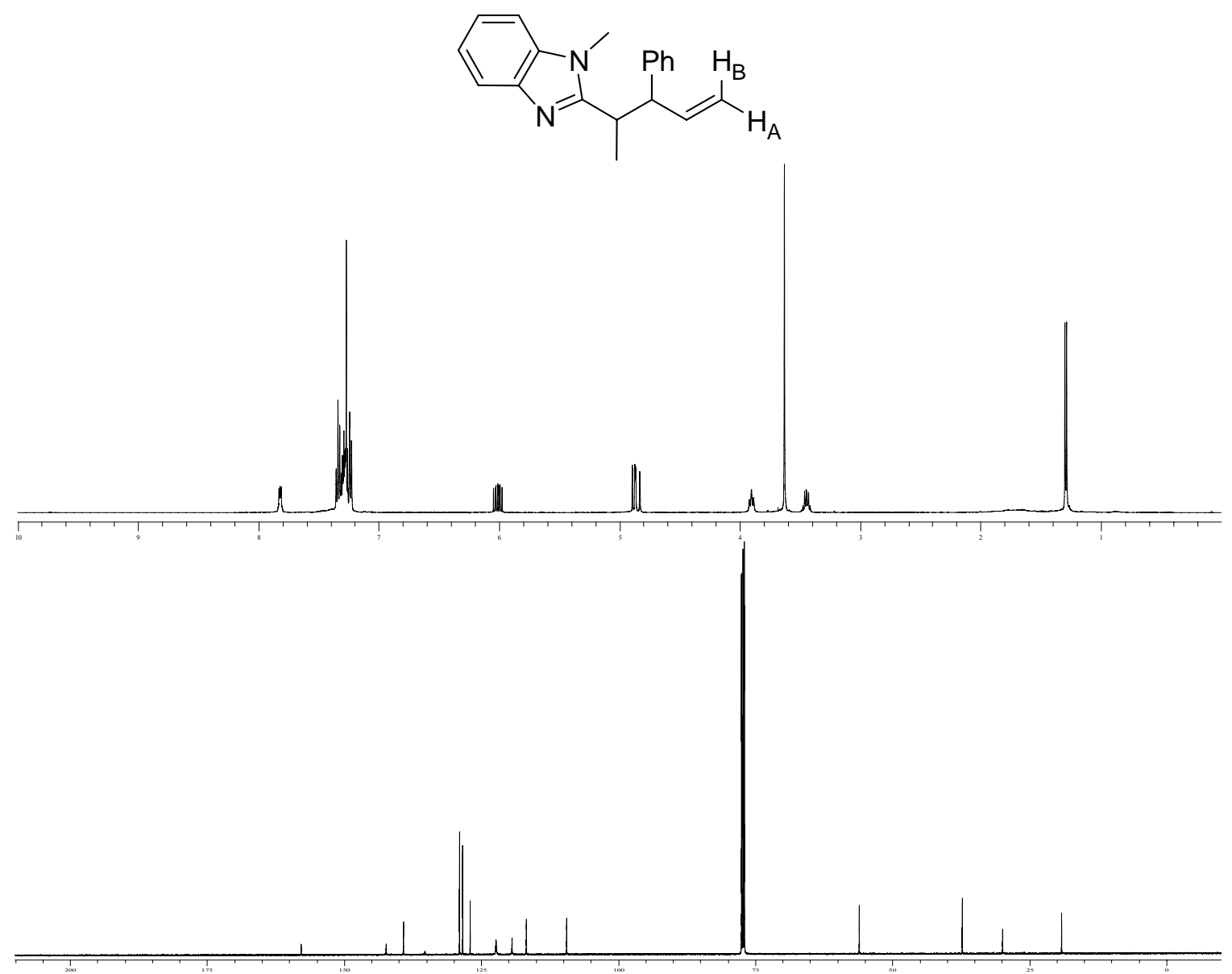

2e: (1-methyl-2-(3-phenylpent-4-en-2-yl)-1H-benzimidazole): yellow solid: 95:5 to 90:10 hex/EtOAc: ${ }^{1} \mathrm{H}$ NMR $\left(500 \mathrm{MHz}, \mathrm{CDCl}_{3}\right) \delta 1.29\left(\mathrm{~d}, 3 \mathrm{H}: J=6.9 \mathrm{~Hz}, \mathrm{R}_{2} \mathrm{CHCH}_{3}\right), 3.44$ (dq, $\left.1 \mathrm{H}: J=6.9,9.5 \mathrm{~Hz}, \mathrm{R}_{2} \mathrm{CHCH}_{3}\right), 3.63\left(\mathrm{~s}, 3 \mathrm{H}: \mathrm{NCH}_{3}\right), 3.90$ (t, $1 \mathrm{H}: J=9.5 \mathrm{~Hz}$, $\mathrm{R}_{2} \mathrm{CHPh}$ ), 4.84 (app. d, $\left.1 \mathrm{H}: J=17.0 \mathrm{~Hz}, \mathrm{H}_{\mathrm{B}}\right), 4.88$ (d, $1 \mathrm{H}: J=0.7,10.4 \mathrm{~Hz}, \mathrm{H}_{\mathrm{A}}$ ), 6.01 (ddd, 1H: $J=8.2,10.4,17.0 \mathrm{~Hz}, \mathrm{RCH}=\mathrm{CH}_{2}$ ), 7.24 (dd, 2H: $J=7.8,9.0$. Hz, Ar H's), 7.28 (m, 4H: Ar CH), 7.33 (overlapping dd, 3H: $J=7.8,15.3 \mathrm{~Hz}, \mathrm{Ar} \mathrm{CH}^{\prime}$ ); ${ }^{13} \mathrm{C} \mathrm{NMR}$ $\left(125 \mathrm{MHz}, \mathrm{CDCl}_{3}\right) \delta 19.1\left(\mathrm{R}_{2} \mathrm{CHCH}_{3}\right), 29.9\left(\mathrm{NCH}_{3}\right), 37.2\left(\mathrm{R}_{2} \mathrm{CHCH}_{3}\right), 56.0\left(\mathrm{R}_{2} \mathrm{CHPh}\right)$, 109.4 (Ar CH), $116.8\left(\mathrm{RCH}=\mathrm{CH}_{2}\right), 119.4(\mathrm{Ar} \mathrm{CH}), 122.3(\mathrm{Ar} \mathrm{CH}), 127.0(\mathrm{Ar} \mathrm{CH}), 128.7$ (2 Ar CH's), 128.9 (2 Ar CH's), 135.2 (quat $\mathrm{Ar} \mathrm{C}$ ), $139.2\left(\mathrm{RCH}=\mathrm{CH}_{2}\right), 142.3$ (quat. Ar C), $157.8(\mathrm{NC}=\mathrm{N})$; IR $\left(\mathrm{CH}_{2} \mathrm{Cl}_{2}\right) v_{\max }: 3054,2987,1599,1550,1422,1265,1153,896$; HRMS calcd for $[\mathrm{M}+\mathrm{H}] 277.1705$, found 277.1698. 


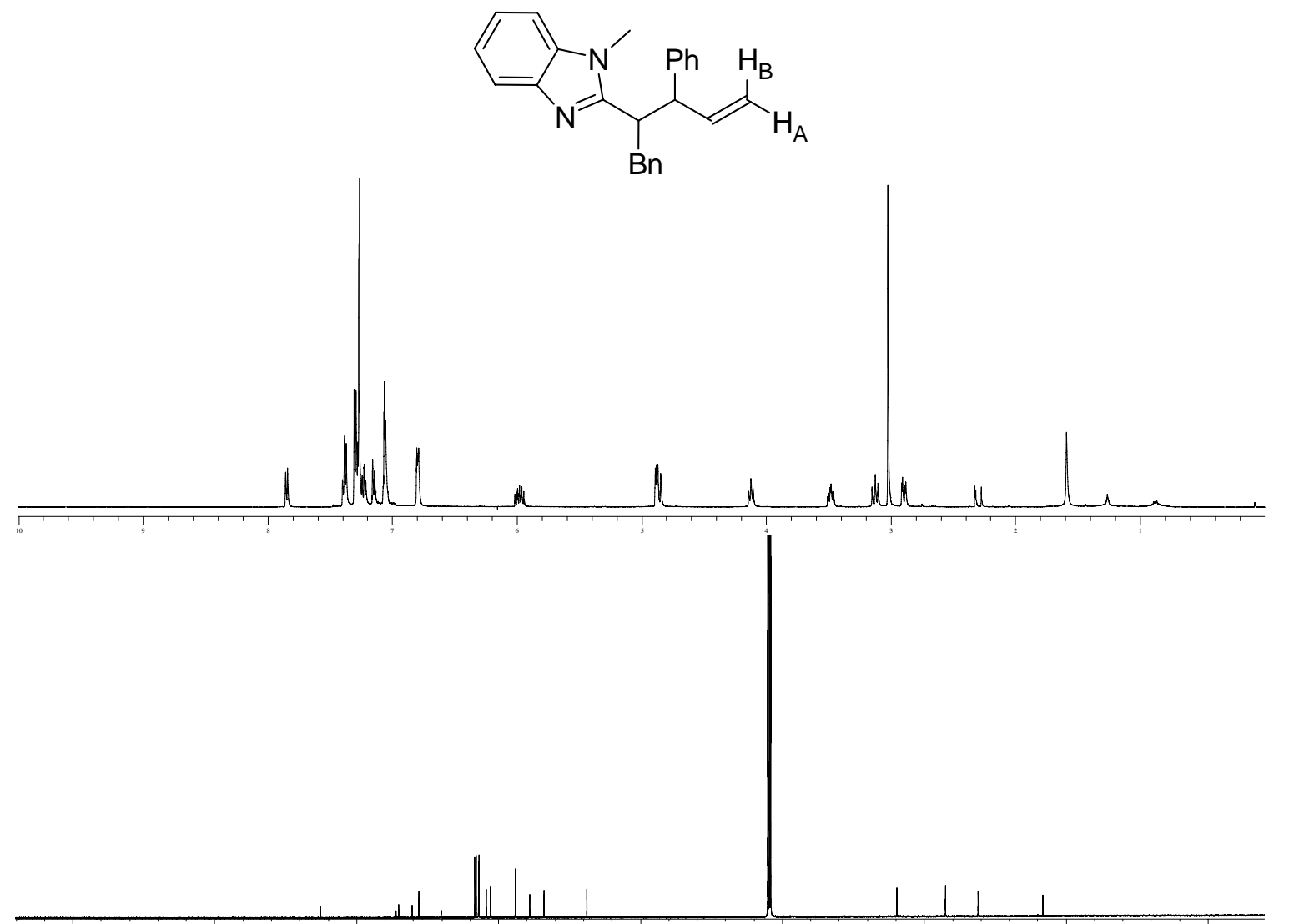

2f: (2-(1,3-diphenylpent-4-en-2-yl)-1-methyl-1H-benzimidazole): pale yellow oil: 95:5 hex/EtOAc: ${ }^{1} \mathrm{H}$ NMR (500 MHz, $\left.\mathrm{CDCl}_{3}\right) \delta 2.90$ (dd, 1H: $\left.J=3.8,13.1 \mathrm{~Hz}, \mathrm{PhCHH}\right), 3.02$ (s, $3 \mathrm{H}: \mathrm{NCH}_{3}$ ), 3.12 (dd, $\left.1 \mathrm{H}: J=11.2,13.1 \mathrm{~Hz}, \mathrm{PhCHH}\right), 3.48$ (dt, $1 \mathrm{H}: J=4.1,10.2 \mathrm{~Hz}$, $\mathrm{R}_{2} \mathrm{CHBn}$ ), 4.12 (t, $1 \mathrm{H}: J=8.5 \mathrm{~Hz}, \mathrm{R}_{2} \mathrm{CHPh}$ ), 4.86 (app. d, $1 \mathrm{H}: J=17.0 \mathrm{~Hz}, \mathrm{H}_{\mathrm{B}}$ ), 4.89 (app. d, 1H: $J=10.3 \mathrm{~Hz}, \mathrm{H}_{\mathrm{A}}$ ), 5.98 (ddd, 1H: $\left.J=8.1,10.3,17.0 \mathrm{~Hz}, \mathrm{RCH}=\mathrm{CH}_{2}\right), 6.79$ (m, 2H: overlapping Ar CH's), 7.06 (m, 3H: overlapping Ar CH's), 7.15 (d, 1H: J= 7.9 $\mathrm{Hz}$, Het Ar CH), 7.23 (dt, 1H: 1.1, 7.9 Hz, Het Ar CH), 7.26 (m, 1H: Het Ar CH), 7.30 (m, 3H: overlapping Ar CH's), 7.39 (m, 2H: overlapping Ar CH's), 7.85 (d, 1H: J= 7.9 $\mathrm{Hz}$, Het Ar CH); ${ }^{13} \mathrm{C}$ NMR $\left(125 \mathrm{MHz}, \mathrm{CDCl}_{3}\right) \delta 29.0\left(\mathrm{NCH}_{3}\right), 40.4\left(\mathrm{CH}_{2} \mathrm{Ph}\right), 46.2$ $\left(\mathrm{R}_{2} \mathrm{CHBn}\right), 54.8\left(\mathrm{R}_{2} \mathrm{CHPh}\right), 109.4(\mathrm{Ar} \mathrm{CH}), 116.9\left(\mathrm{RCH}=\mathrm{CH}_{2}\right), 119.4(\mathrm{Ar} \mathrm{CH}), 122.0$ (overlapping Ar CH's), $126.4(\mathrm{Ar} \mathrm{CH}), 127.1$ (Ar CH), $128.4(\mathrm{Ar} \mathrm{CH}), 128.5(\mathrm{Ar} \mathrm{CH})$, 128.9 (Ar CH), 129.1 (Ar CH), 135.0 (quat. Ar C), $139.0\left(\mathrm{RCH}=\mathrm{CH}_{2}\right), 140.2$ (quat. $\mathrm{Ar}$ C), 142.5 (quat. $\mathrm{Ar} \mathrm{CH}$ ), 143.0 (quat. $\mathrm{Ar} \mathrm{C}$ ), $156.3(\mathrm{NC}=\mathrm{N})$; IR $\left(\mathrm{CH}_{2} \mathrm{Cl}_{2}\right)$ v $\max$ : 3054, 2987, 1601, 1551, 1493, 1466, 1440, 1422, 1267, 1154, 896; HRMS calcd for [M+H] 353.2018, found 353.2015. 


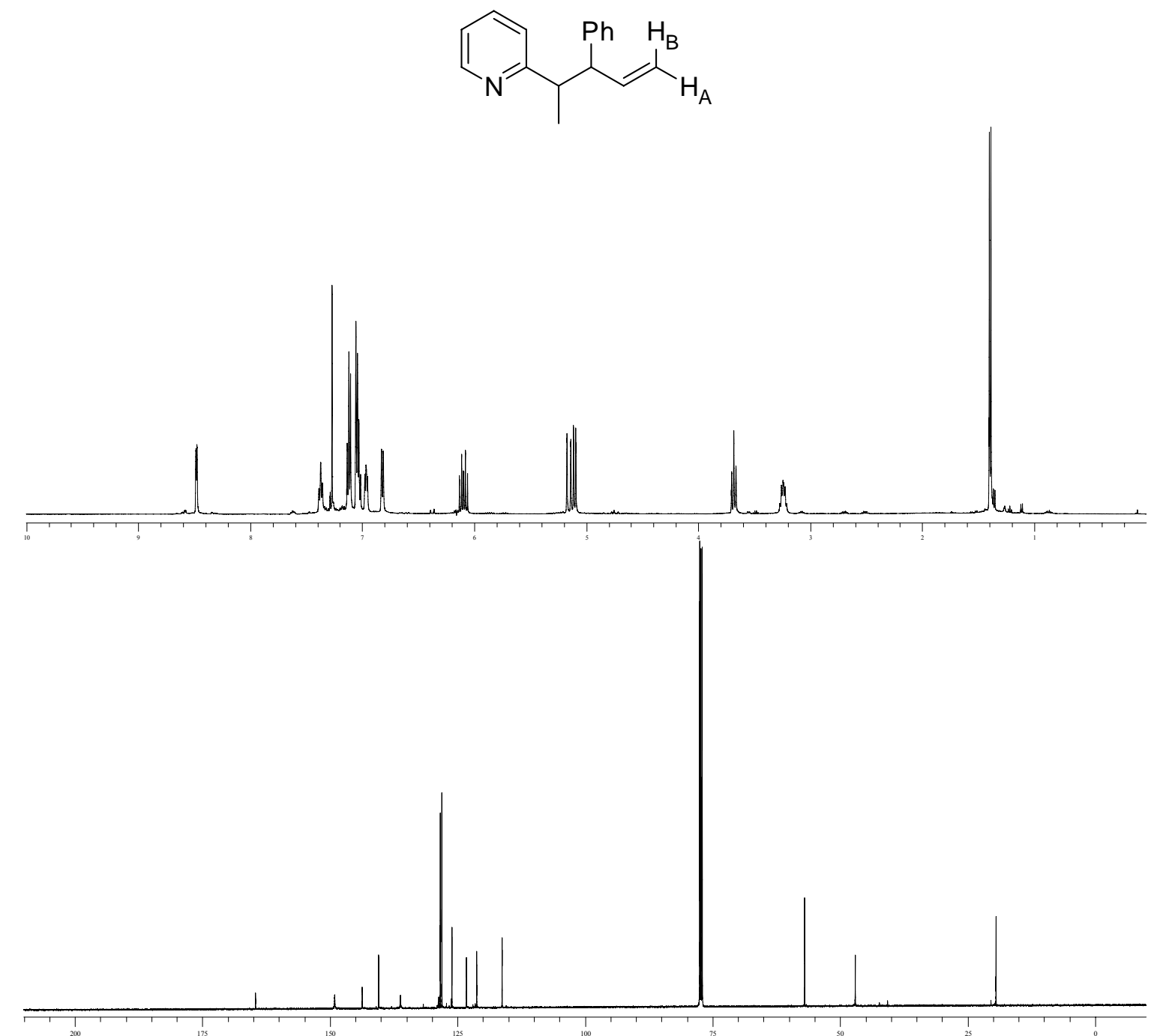

2g: (2-(2-phenylpent-4-en-2-yl)pyridine): pale orange oil: 95:5 hex/EtOAc: ${ }^{1} \mathrm{H}$ NMR $\left(500 \mathrm{MHz}, \mathrm{CDCl}_{3}\right) \delta 1.39$ (d, 3H: $\left.J=7.0 \mathrm{~Hz}, \mathrm{R}_{2} \mathrm{CHCH}_{3}\right), 3.24$ (dq, $1 \mathrm{H}: J=7.0,9.7 \mathrm{~Hz}$, $\mathrm{R}_{2} \mathrm{CHCH}_{3}$ ), $3.68\left(\mathrm{t}, 1 \mathrm{H}: J=9.7 \mathrm{~Hz}, \mathrm{R}_{2} \mathrm{CHPh}\right), 5.10\left(\mathrm{dd}, 1 \mathrm{H}: J=1.4,10.1 \mathrm{~Hz}, \mathrm{H}_{\mathrm{A}}\right), 5.16$ (app. d, 1H: $\left.J=17.0 \mathrm{~Hz}, \mathrm{H}_{\mathrm{B}}\right), 6.10\left(\mathrm{dt}, 1 \mathrm{H}: J=9.7,17.0 \mathrm{~Hz}, \mathrm{RCH}=\mathrm{CH}_{2}\right), 6.82(\mathrm{~d}, 1 \mathrm{H}: J$ $=7.7 \mathrm{~Hz}, \mathrm{R}(\mathrm{C}=\mathrm{N}) \mathrm{CH}), 6.96(\mathrm{dd}, 1 \mathrm{H}: J=4.4 \mathrm{~Hz}, \mathrm{NCH}=\mathrm{CH}), 7.04(\mathrm{~m}, 3 \mathrm{H}:$ Ar CH's), 7.12 (m, 2H: Ar CH's), 7.37 (t, 1H: J= $7.5 \mathrm{~Hz}, \mathrm{NCH}=\mathrm{CHCH}), 8.48$ (d, $1 \mathrm{H}: J=4.4 \mathrm{~Hz}$, $\mathrm{NCH}) ;{ }^{13} \mathrm{C} \mathrm{NMR}\left(125 \mathrm{MHz}, \mathrm{CDCl}_{3}\right) \delta 19.4\left(\mathrm{R}_{2} \mathrm{CHCH}_{3}\right), \quad 47.0\left(\mathrm{R}_{2} \mathrm{CHCH}_{3}\right), 56.9$ $\left(\mathrm{R}_{2} \mathrm{CHPh}\right), 116.3\left(\mathrm{RCH}=\mathrm{CH}_{2}\right), 121.2(\mathrm{NCH}=\mathrm{CH}), 123.3(\mathrm{~N}=\mathrm{CRCH}), 126.1(\mathrm{Ar} \mathrm{CH})$, $128.1(\mathrm{Ar} \mathrm{CH}), 128.7(\mathrm{Ar} \mathrm{CH}), 136.2(\mathrm{NCH}=\mathrm{CHCH}), 140.4\left(\mathrm{RCH}=\mathrm{CH}_{2}\right), 143.7$ (quat. Ar C), $149.1(\mathrm{NCH}), 164.6(\mathrm{~N}=\mathrm{CR})$; IR $\left(\mathrm{CH}_{2} \mathrm{Cl}_{2}\right) v_{\max }$ : 3054, 2987, 1637, 1591, 1569, 1550, 1474, 1422, 1263, 1150, 992, 896; HRMS calcd for [M+H] 224.1439, found 224.1426. 


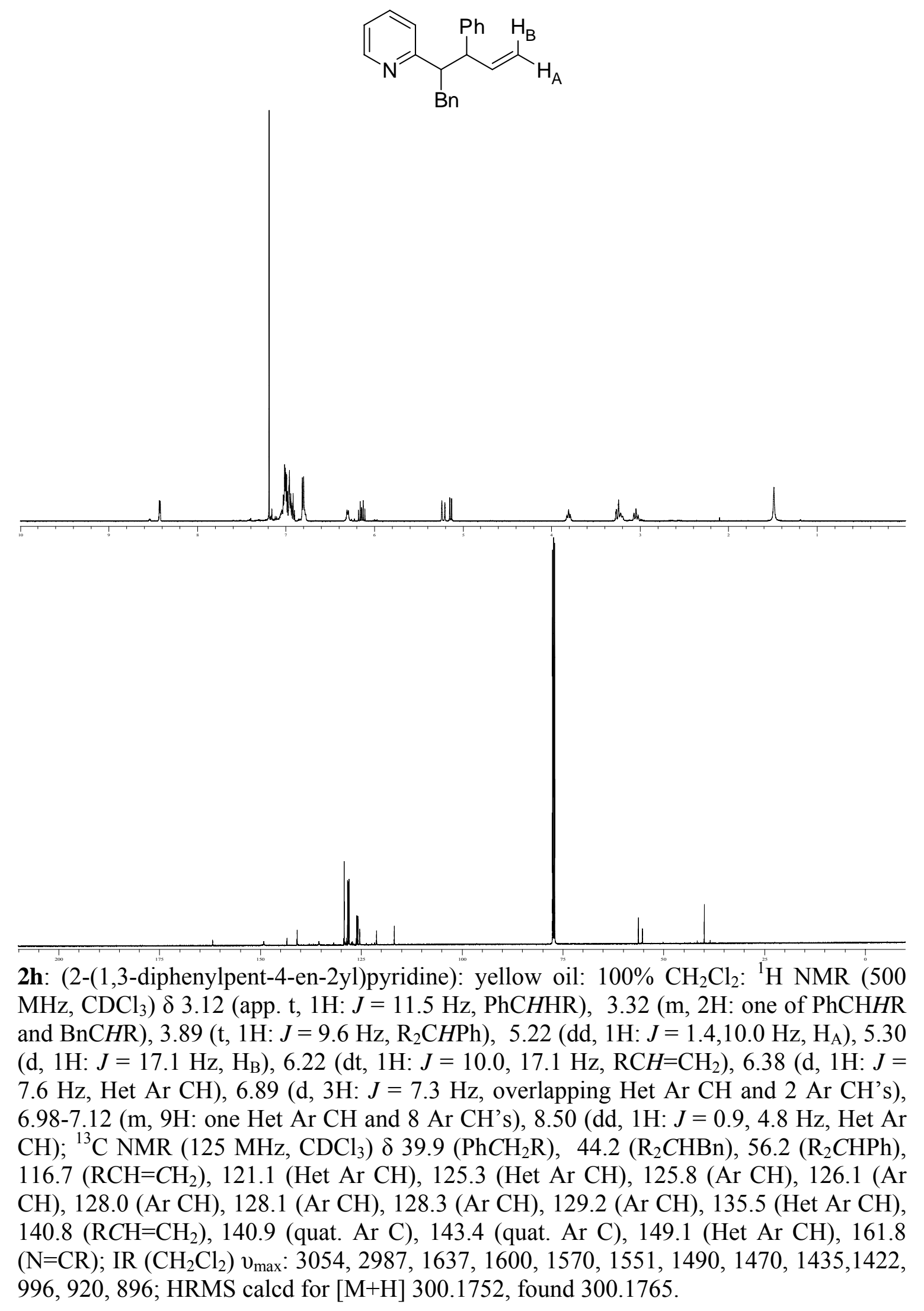




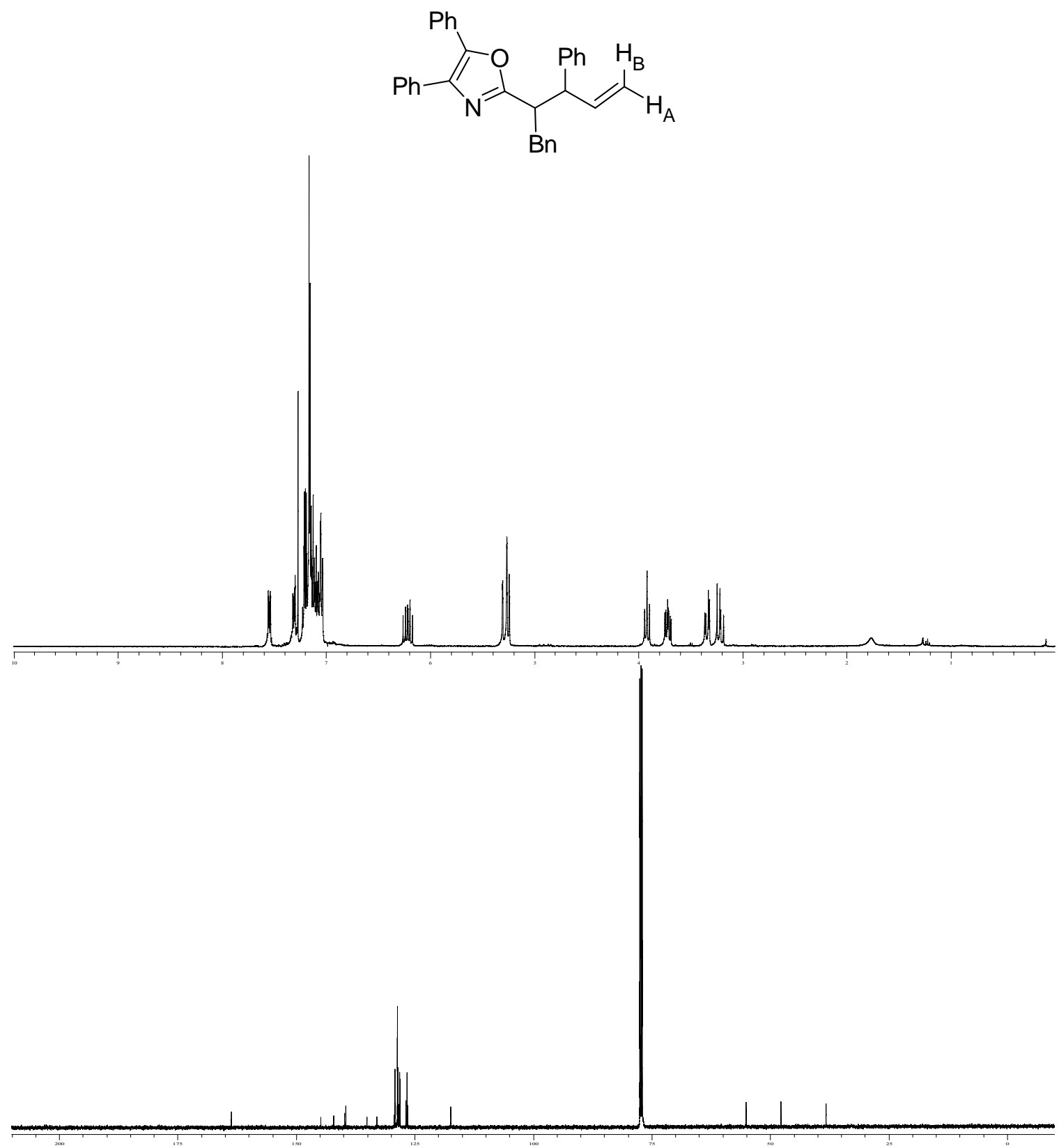

2i: (2-(1,3-diphenylpent-4-en-2-yl)-4,5-diphenyloxazole): yellow oil: 98:2 hex/EtOAc: ${ }^{1} \mathrm{H}$ NMR (400 MHz, $\mathrm{CDCl}_{3}$ ) $\delta 3.20$ (dd, $\left.1 \mathrm{H}: J=10.8,13.9 \mathrm{~Hz}, \mathrm{RCHHPh}\right), 3.31$ (dd, $1 \mathrm{H}:$ $J=4.2,13.9 \mathrm{~Hz}, \mathrm{RCHHPh}), 3.61$ (ddd, $1 \mathrm{H}: J=4.2,10.8,10.8 \mathrm{~Hz}, \mathrm{R}_{2} \mathrm{CHCH}_{2} \mathrm{Ph}$ ), 3.87 (t, $1 \mathrm{H}: J=9.4 \mathrm{~Hz}, \mathrm{R}_{2} \mathrm{CHPh}$ ), 5.26 (dd, $1 \mathrm{H}: J=1.3,9.8 \mathrm{~Hz}, \mathrm{H}_{\mathrm{A}}$ ), 5.29 (app. d, $1 \mathrm{H}: J=17.0$ $\mathrm{Hz}, \mathrm{H}_{\mathrm{B}}$ ), 6.25 (dt, 1H: $J=9.8,17.0 \mathrm{~Hz}, \mathrm{RCH}=\mathrm{CH}_{2}$ ), 7.12-7.17 (m, 4H: Ar CH's), 7.197.25 (m, 8H: Ar CH's), 7.27-7.34 (m, 6H: Ar CH's), 7.46 (m, 2H: Ar CH's); ${ }^{13} \mathrm{C}$ NMR $\left(125 \mathrm{MHz}, \mathrm{CDCl}_{3}\right) \delta 38.4\left(\mathrm{RCH}_{2} \mathrm{Ph}\right), 48.0\left(\mathrm{R}_{2} \mathrm{CHCH}_{2} \mathrm{Ph}\right), 55.4\left(\mathrm{R}_{2} \mathrm{CHPh}\right), 118.1$ $\left(\mathrm{RCH}=\mathrm{CH}_{2}\right), 127.2(\mathrm{Ar} \mathrm{CH}), 127.4(\mathrm{Ar} \mathrm{CH}), 127.6(\mathrm{Ar} \mathrm{CH}), 128.8(\mathrm{Ar} \mathrm{CH}), 128.9(\mathrm{Ar}$ $\mathrm{CH}), 128.9$ (Ar CH), 129.1 (Ar CH), 129.3 (Ar CH), 129.4 (Ar CH), 129.6 (overlapping Ar CH's), 129.9 (Ar CH), 130.1 (quat. Ar C), 133.8 (quat. Ar C), 135.9 (quat. Ar C), $140.3\left(\mathrm{RCH}=\mathrm{CH}_{2}\right), 140.6$ (quat. Ar C), 142.9 (quat. Ar C), 145.7 (quat. Ar C), 164.7 
$(\mathrm{N}=\mathrm{CO}) ; \mathrm{IR}\left(\mathrm{CH}_{2} \mathrm{Cl}_{2}\right) v_{\max }: 3054,2986,1695,1602,1560,1494,1436,1421,1263,1157$, 1072, 1025, 992, 963, 896; HRMS calcd for [M+H] 442.2171, found 442.2175.
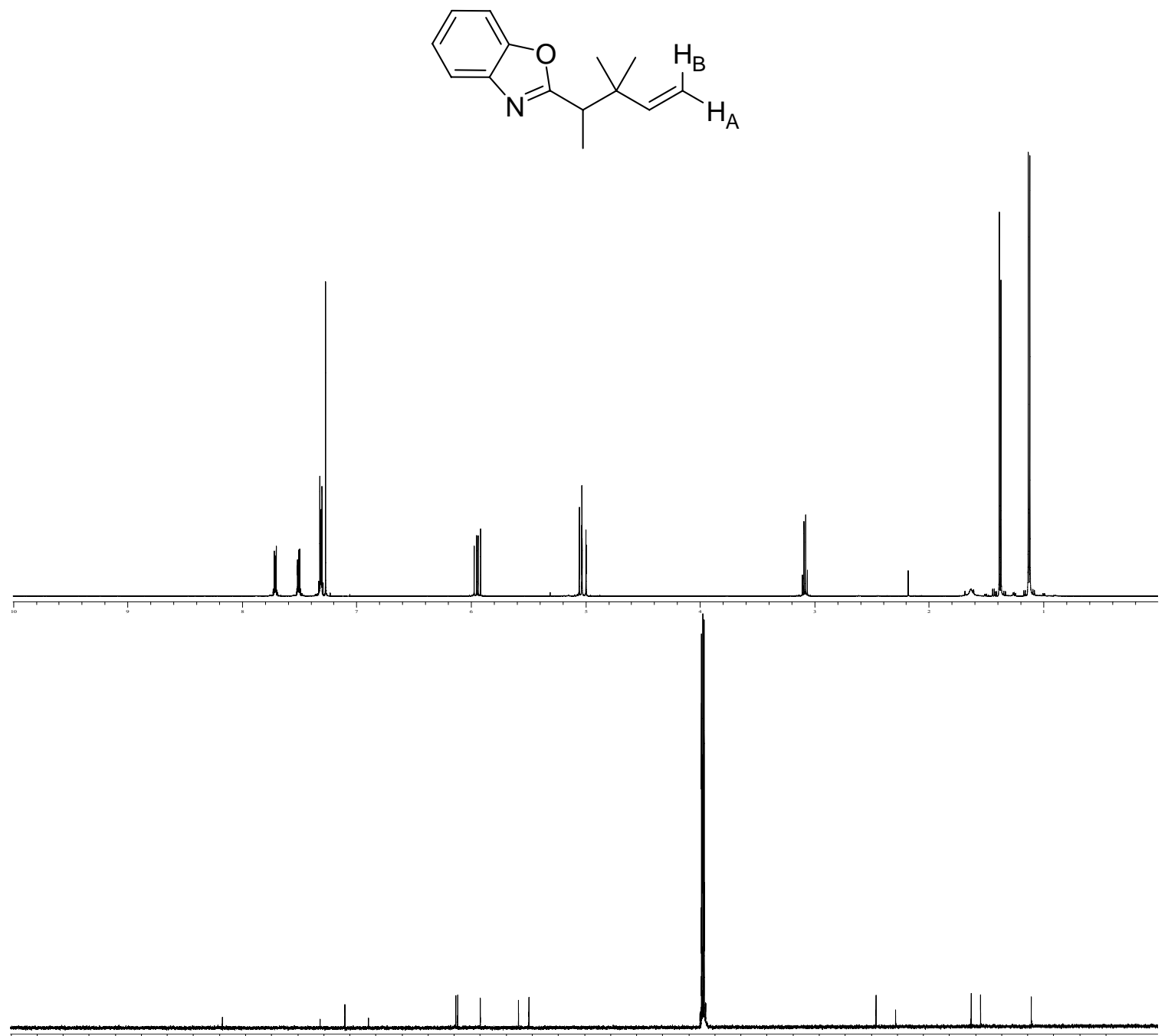

2j: (2-(3,3-dimethylpent-4-en-2-yl)benzoxazole): pale orange oil: 98:2 hex/EtOAc: ${ }^{1} \mathrm{H}$

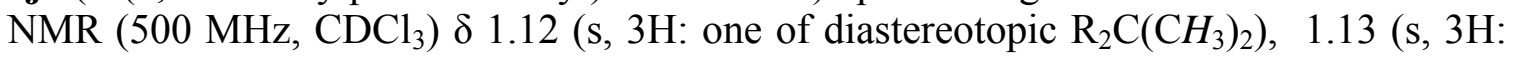
one of diastereotopic $\left.\mathrm{R}_{2} \mathrm{C}\left(\mathrm{CH}_{3}\right)_{2}\right), 1.38$ (d, $3 \mathrm{H}: J=7.2 \mathrm{~Hz}, \mathrm{R}_{2} \mathrm{CHCH}_{3}$ ), 3.08 (q, $1 \mathrm{H}: J=$ $7.2 \mathrm{~Hz}, \mathrm{R}_{2} \mathrm{CHCH}_{3}$ ), 5.01 (dd, $1 \mathrm{H}: J=1.1,17.5 \mathrm{~Hz}, \mathrm{H}_{\mathrm{B}}$ ), 5.04 (dd, $1 \mathrm{H}: J=1.1,10.8 \mathrm{~Hz}$, $\left.\mathrm{H}_{\mathrm{A}}\right), 5.94\left(\mathrm{dd}, 1 \mathrm{H}: J=10.8,17.5 \mathrm{~Hz}, \mathrm{RCH}=\mathrm{CH}_{2}\right), 7.31(\mathrm{~m}, 2 \mathrm{H}: \mathrm{Het} \mathrm{Ar} \mathrm{CH}), 7.51(\mathrm{~m}, 1 \mathrm{H}$ : Het $\mathrm{ArCH}), 7.71(\mathrm{~m}, 1 \mathrm{H}$ : Het $\mathrm{ArCH}) ;{ }^{13} \mathrm{C}$ NMR $\left(125 \mathrm{MHz}, \mathrm{CDCl}_{3}\right) \delta 14.5\left(\mathrm{R}_{2} \mathrm{CHCH}_{3}\right)$, 24.0 (one of diastereotopic $\left.\mathrm{R}_{2} \mathrm{C}\left(\mathrm{CH}_{3}\right)_{2}\right), 25.8$ (one of diastereotopic $\left.\mathrm{R}_{2} \mathrm{C}\left(\mathrm{CH}_{3}\right)_{2}\right), 40.3$ $\left(\mathrm{R}_{2} \mathrm{C}\left(\mathrm{CH}_{3}\right)_{2}\right), 44.0\left(\mathrm{R}_{2} \mathrm{CHCH}_{3}\right), 110.6(\mathrm{Het} \mathrm{Ar} \mathrm{CH}), 112.6\left(\mathrm{RCH}=\mathrm{CH}_{2}\right), 119.9(\mathrm{Het} \mathrm{Ar}$ $\mathrm{CH}), 124.4$ (Het $\mathrm{Ar} \mathrm{CH}), 124.6$ (Het $\mathrm{Ar} \mathrm{CH}), 141.4$ (quat. Het $\mathrm{Ar} \mathrm{C}), 145.9\left(\mathrm{RCH}=\mathrm{CH}_{2}\right)$, 150.6 (quat. Het $\mathrm{Ar} \mathrm{C}), 169.4(\mathrm{~N}=\mathrm{CO})$; IR $\left(\mathrm{CH}_{2} \mathrm{Cl}_{2}\right) v_{\max }$ : 3054, 2984, 1782, 1638, 1610, $1563,1456,1421,1378,1263,1139,1074,1003,922,895$; HRMS calcd for $[\mathrm{M}+\mathrm{H}]$ 216.1388 , found 216.1390 . 


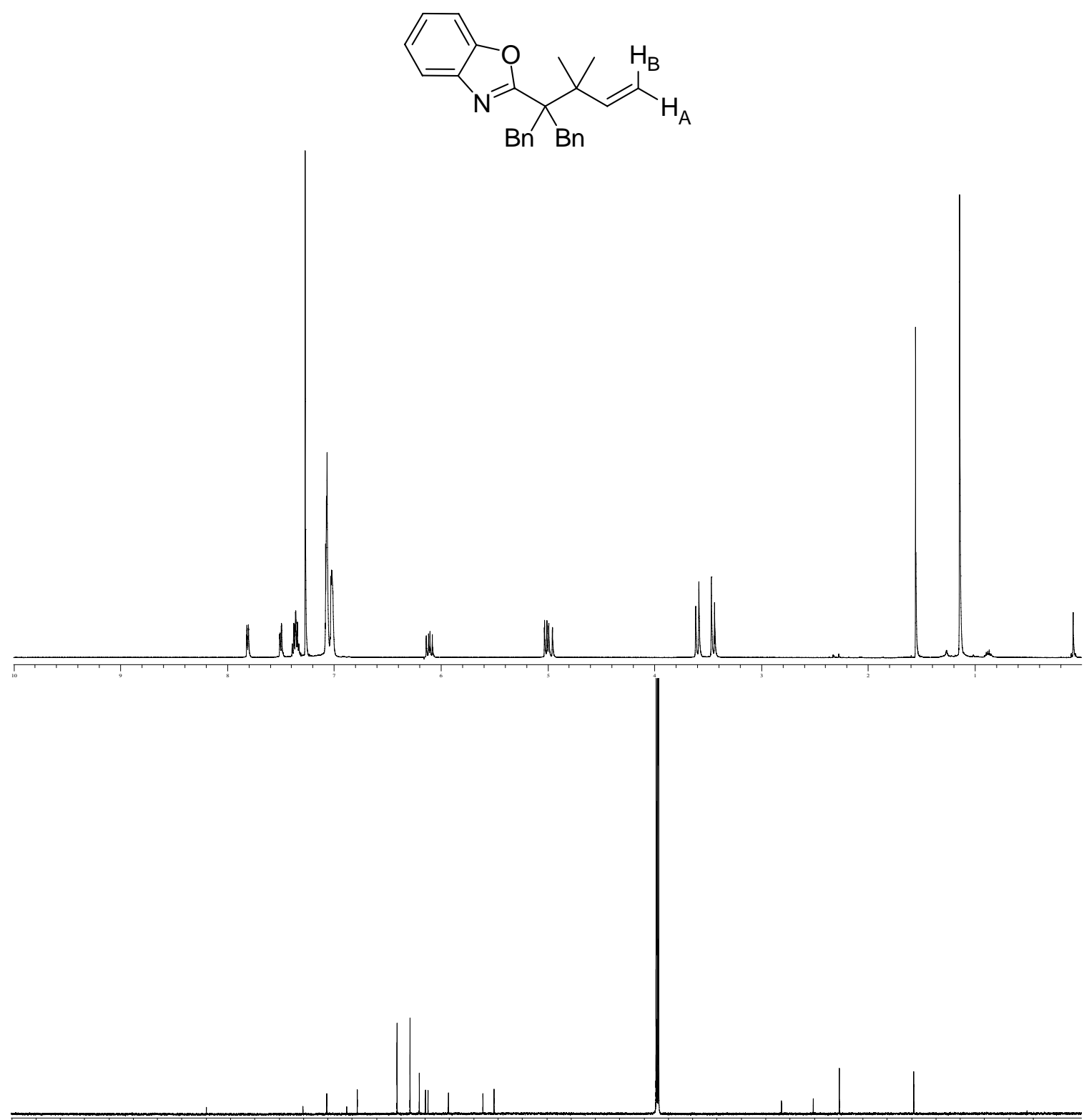

2k: (2-(2-benzyl-3,3-dimethyl-1-phenylpent-4-en-2-yl)benzoxazole): pale yellow solid: 98:2 hex/EtOAc: ${ }^{1} \mathrm{H}$ NMR $\left(500 \mathrm{MHz}, \mathrm{CDCl}_{3}\right) \delta 1.14$ (s, 6H: $\left.\mathrm{R}_{2} \mathrm{C}\left(\mathrm{CH}_{3}\right)_{2}\right), 3.45$ (d, 2H: $J$ $\left.=14.9 \mathrm{~Hz}, \mathrm{R}_{2} \mathrm{C}(\mathrm{CHHPh})_{2}\right), 3.60\left(\mathrm{~d}, 2 \mathrm{H}: J=14.9 \mathrm{~Hz}, \mathrm{R}_{2} \mathrm{C}(\mathrm{CHHPh})_{2}\right), 4.97(\mathrm{dd}, 1 \mathrm{H}: J=$ 1.0, $\left.17.4 \mathrm{~Hz}, \mathrm{H}_{\mathrm{B}}\right), 5.02$ (dd, $\left.1 \mathrm{H}: J=1.0,10.2 \mathrm{~Hz}, \mathrm{H}_{\mathrm{A}}\right), 6.11(\mathrm{dd}, 1 \mathrm{H}: J=10.2,17.4 \mathrm{~Hz}$, $\mathrm{RCH}=\mathrm{CH}_{2}$ ), 7.02 (m, 4H: Ar CH's), 7.07 (m, 6H: Ar CH's), 7.36 (dp, 2H: $J=1.5,7.3$ Hz, Ar CH's), 7.50 (app. d, 1H: $J=7.3 \mathrm{~Hz}$, Het Ar CH), 7.81 (app d., 1H: $J=7.3 \mathrm{~Hz}$, Het

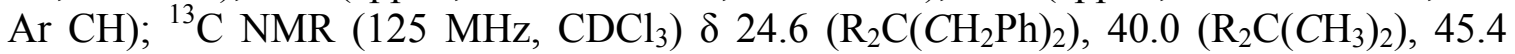
$\left(\mathrm{R}_{2} \mathrm{C}\left(\mathrm{CH}_{3}\right)_{2}\right), 52.0\left(\mathrm{R}_{2} \mathrm{C}\left(\mathrm{CH}_{2} \mathrm{Ph}\right)_{2}\right), 111.4(\mathrm{Het} \mathrm{Ar} \mathrm{CH}), 113.7\left(\mathrm{RCH}=\mathrm{CH}_{2}\right), 120.9(\mathrm{Het} \mathrm{Ar}$ $\mathrm{CH}), 125.1$ (Het $\mathrm{Ar} \mathrm{CH}), 125.6$ (Het $\mathrm{Ar} \mathrm{CH}), 126.9$ (Ar CH), 128.8 (Ar CH), 131.5 (Ar $\mathrm{CH}$ ), 139.7 (quat. $\mathrm{Ar} \mathrm{C}$ ), 141.9 (quat. $\mathrm{Ar} \mathrm{C}$ ), $146.0\left(\mathrm{RCH}=\mathrm{CH}_{2}\right), 151.0$ (quat. $\mathrm{Ar} \mathrm{C}$ ), $170.9(\mathrm{~N}=\mathrm{CO})$; IR $\left(\mathrm{CH}_{2} \mathrm{Cl}_{2}\right) v_{\max }: 3054,2987,1603,1550,1496,1456,1421,1261,1154$, 896; HRMS calcd for [M+H] 382.2171, found 382.2172. 


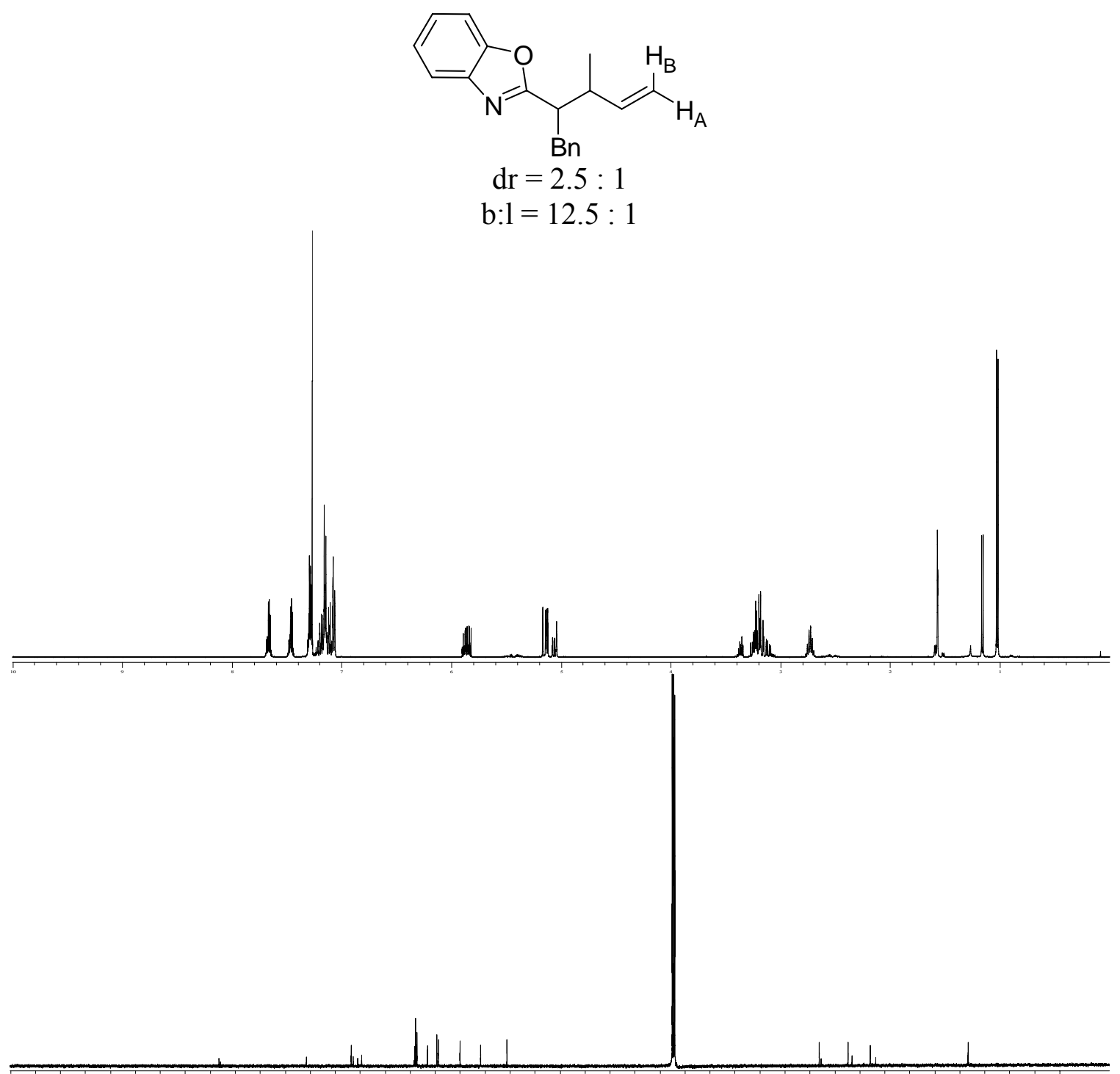

2l: (2-(3-methyl-1-phenylpent-4-en-2-yl)benzoxazole): colorless oil: 99:1 hex/EtOAc: ${ }^{1} \mathrm{H}$ NMR $\left(500 \mathrm{MHz}, \mathrm{CDCl}_{3}\right) \delta$ major diastereomer: $1.02(\mathrm{~d}, 3 \mathrm{H}: \quad J=7.1 \mathrm{~Hz}$, $\mathrm{CH}_{2}=\mathrm{CHCHCH}_{3}$ ), 2.73 (sext., $1 \mathrm{H}: J=7.1 \mathrm{~Hz}, \mathrm{CH}_{2}=\mathrm{CHCHCH}_{3}$ ), 3.15-3.22 (m, 3H: overlapping $\mathrm{PhCH}_{2}$ and $\left.\mathrm{PhCH}_{2} \mathrm{CHR}_{2}\right), 5.13\left(\mathrm{dd}, 1 \mathrm{H}: J=1.1,10.2 \mathrm{~Hz}, \mathrm{H}_{\mathrm{A}}\right), 5.15(\mathrm{dd}, 1 \mathrm{H}$ : $\left.J=1.1,17.2 \mathrm{~Hz}, \mathrm{H}_{\mathrm{B}}\right), 5.86$ (ddd, $\left.1 \mathrm{H}: J=8.3,10.2,17.2 \mathrm{~Hz}, \mathrm{RCH}=\mathrm{CH}_{2}\right), 7.07$ (m, 2H: overlapping Ar CH's), 7.20 (m, 3H: overlapping Ar CH's), 7.29 (m, 2H: overlapping Het Ar CH's), 7.46 (m, 1H: Het $\mathrm{Ar} \mathrm{CH}), 7.67$ (m, 1H: Het $\mathrm{Ar} \mathrm{CH})$; minor diastereomer: 1.16 (d, 3H: $J=7.1 \mathrm{~Hz}, \mathrm{CH}_{2}=\mathrm{CHCHCH}_{3}$ ), 2.73 (sext., $1 \mathrm{H}: J=7.1 \mathrm{~Hz}, \mathrm{CH}_{2}=\mathrm{CHCHCH}_{3}$ ), 3.11 (m, 1H: PhCHHR), 3.25 (m, 1H: $\mathrm{BnCHR}_{2}$ ), 3.36 (dt, 1H: $J=5.6,9.4 \mathrm{~Hz}, \mathrm{PhCHHR}$ ), 5.06 (m, 2H: $\mathrm{RCH}=\mathrm{CH}_{2}$ ), 5.87 (ddd, $\left.1 \mathrm{H}: 8.2,10.4,17.0 \mathrm{~Hz}, \mathrm{CH}_{2}=\mathrm{CHR}\right), 7.12$ (m, 2H: overlapping Ar CH's), 7.20 (m, 3H: overlapping Ar CH's), 7.29 (m, 2H: overlapping Het Ar CH's), 7.46 (m, 1H: Het Ar CH), 7.67 (m, 1H: Het Ar CH); ${ }^{13} \mathrm{C}$ NMR (125 MHz, $\left.\mathrm{CDCl}_{3}\right) \delta$ major diastereomer: $18.3\left(\mathrm{R}_{2} \mathrm{CHCH}_{3}\right), 37.9\left(\mathrm{PhCH}_{2} \mathrm{R}\right), 42.3\left(\mathrm{CH}_{2}=\mathrm{CHCHCH}_{3}\right)$, $48.1\left(\mathrm{PhCH}_{2} \mathrm{CHR}_{2}\right), 110.6(\mathrm{Het} \mathrm{Ar} \mathrm{CH}), 115.8\left(\mathrm{RCH}=\mathrm{CH}_{2}\right), 120.0($ Het $\mathrm{Ar} \mathrm{CH}), 124.3$ (Het Ar CH), 124.6 (Het Ar CH), 126.4 (Ar CH), 128.6 (Ar CH), 128.9 (Ar CH), 139.6 (quat. $\mathrm{Ar} \mathrm{C}$ ), 141.4 (quat. $\mathrm{Ar} \mathrm{C}$ ), $141.7\left(\mathrm{RCH}=\mathrm{CH}_{2}\right), 150.8$ (quat $\left.\mathrm{Ar} \mathrm{C}\right), 168.2(\mathrm{~N}=\mathrm{CO})$; 
minor diastereomer: $18.4\left(\mathrm{R}_{2} \mathrm{CHCH}_{3}\right), 36.8\left(\mathrm{PhCH}_{2} \mathrm{R}\right), 41.5\left(\mathrm{CH}_{2}=\mathrm{CHCHCH}_{3}\right), 47.7$ $\left(\mathrm{PhCH}_{2} \mathrm{CHR}_{2}\right), 110.6(\mathrm{Het} \mathrm{Ar} \mathrm{CH}), 115.9\left(\mathrm{RCH}=\mathrm{CH}_{2}\right), 120.0(\mathrm{Het} \mathrm{Ar} \mathrm{CH}), 124.2(\mathrm{Het}$ $\mathrm{Ar} \mathrm{CH}), 124.6$ (Het $\mathrm{Ar} \mathrm{CH}), 126.5(\mathrm{Ar} \mathrm{CH}), 128.6(\mathrm{Ar} \mathrm{CH}), 129.0(\mathrm{Ar} \mathrm{CH}), 129.1$ (quat 139.7 (quat. $\mathrm{Ar}$ C), $140.5\left(\mathrm{RCH}=\mathrm{CH}_{2}\right), 141.4$ (quat. $\left.\mathrm{Ar} \mathrm{C}\right), 170.0(\mathrm{~N}=\mathrm{CO}) ; \mathrm{IR}\left(\mathrm{CH}_{2} \mathrm{Cl}_{2}\right)$ $v_{\max }: 3054,2986,1641,1565,1485,1455,1421,1264,1141,1003,923,896$; HRMS calcd for $[\mathrm{M}+\mathrm{H}] 278.1545$, found 278.1544 .
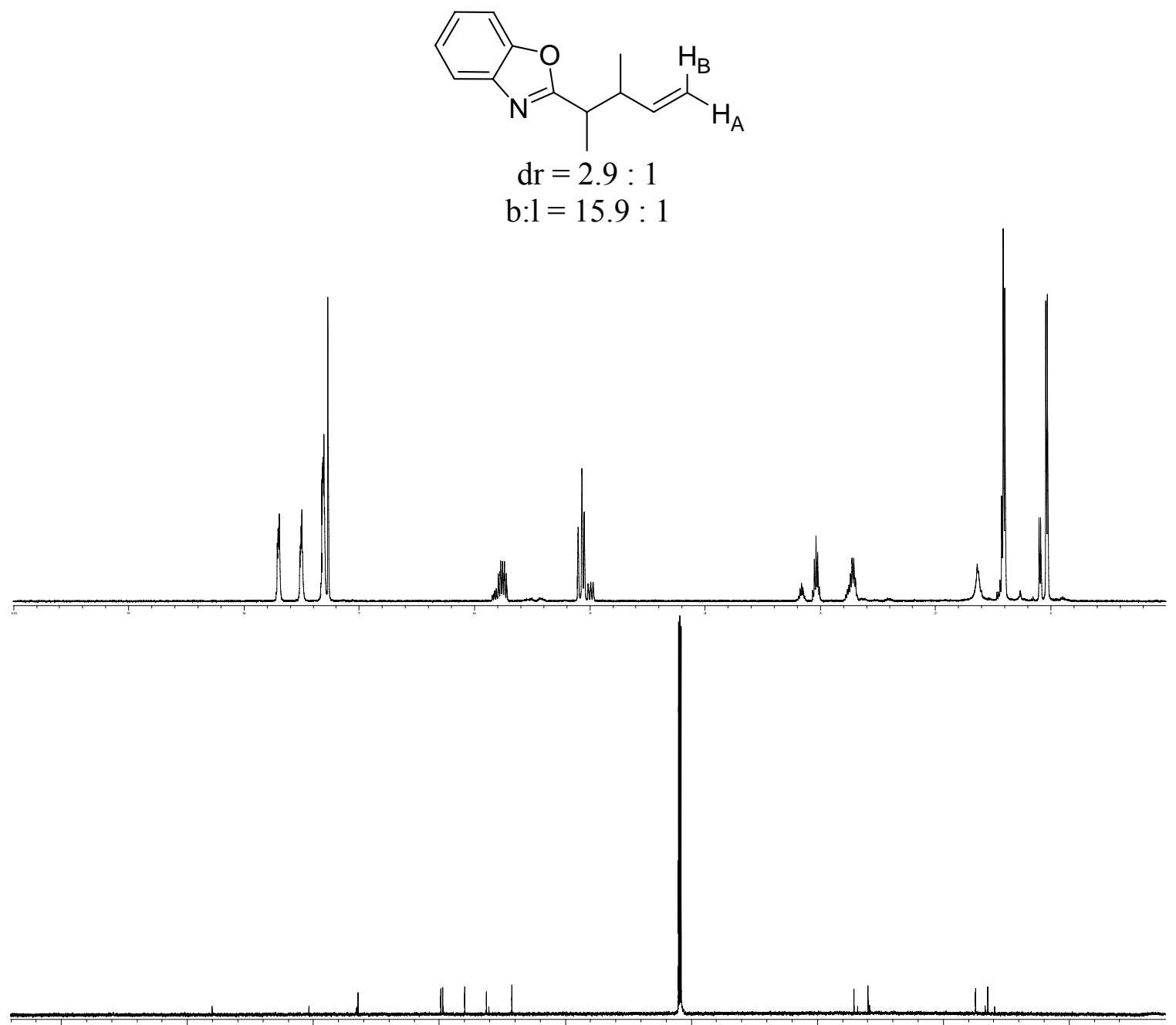

2m: (2-(3-methylpent-4-en-2-yl)benzoxazole): yellow oil: 98:2 hex/EtOAc: ${ }^{1} \mathrm{H}$ NMR $\left(500 \mathrm{MHz}, \mathrm{CDCl}_{3}\right) \delta$ major diastereomer: 1.03 (d, $3 \mathrm{H}: J=6.8 \mathrm{~Hz}, \mathrm{CH}_{2}=\mathrm{CHCHCH}_{3}$ ), 1.40 (d, 3H: $J=7.1 \mathrm{~Hz}, \mathrm{R}_{2} \mathrm{CHCH}_{3}$ ), 2.70 (p, $1 \mathrm{H}: J=7.1 \mathrm{~Hz}, \mathrm{R}_{2} \mathrm{CHCH}_{3}$ ), 3.03 (app. p, $\left.1 \mathrm{H}: J=7.1 \mathrm{~Hz}, \mathrm{CH}_{2}=\mathrm{CHCHCH}_{3}\right), 5.06\left(\mathrm{dd}, 1 \mathrm{H}: J=1.7,10.4 \mathrm{~Hz}, \mathrm{H}_{\mathrm{A}}\right), 5.08(\mathrm{dd}, 1 \mathrm{H}: J=$ 1.0, $17.2 \mathrm{~Hz}, \mathrm{H}_{\mathrm{B}}$ ), $5.76\left(\mathrm{dd}, 1 \mathrm{H}: J=10.4,17.2 \mathrm{~Hz}, \mathrm{RCH}=\mathrm{CH}_{2}\right), 7.31$ (m, $2 \mathrm{H}$ : overlapping Het Ar CH's), 7.50 (m, 1H: Het Ar CH's), 7.70 (m, 1H: Het $\mathrm{Ar} \mathrm{CH}$ ); minor diastereomer: 1.09 (d, 3H: $\left.J=6.8 \mathrm{~Hz}, \mathrm{CH}_{2}=\mathrm{CHCHCH}_{3}\right), 1.42$ (d, 3H: $\left.J=7.1 \mathrm{~Hz}, \mathrm{R}_{2} \mathrm{CHCH}_{3}\right), 2.74$ (p, $1 \mathrm{H}: J=7.1 \mathrm{~Hz}, \mathrm{R}_{2} \mathrm{CHCH}_{3}$ ), 3.16 (app. p, $1 \mathrm{H}: J=7.1 \mathrm{~Hz}, \mathrm{CH}_{2}=\mathrm{CHCHCH}_{3}$ ), 4.99 (m, $2 \mathrm{H}: \mathrm{H}_{\mathrm{B}}$ and $\left.\mathrm{H}_{\mathrm{A}}\right), 5.80\left(\mathrm{~m}, 1 \mathrm{H}: \mathrm{RCH}=\mathrm{CH}_{2}\right), 7.31(\mathrm{~m}, 2 \mathrm{H}$ : overlapping Het Ar CH's), 7.50 (m, 1H: Het Ar CH's), 7.70 (m, 1H: Het $\mathrm{Ar} \mathrm{CH}) ;{ }^{13} \mathrm{C}$ NMR $\left(125 \mathrm{MHz}, \mathrm{CDCl}_{3}\right) \delta$ major diastereomer: $16.1 \quad\left(\mathrm{R}_{2} \mathrm{CHCH}_{3}\right), \quad 18.5 \quad\left(\mathrm{CH}_{2}=\mathrm{CHCHCH}_{3}\right), 39.9 \quad\left(\mathrm{R}_{2} \mathrm{CHCH}_{3}\right), 42.7$ $\left(\mathrm{CH}_{2}=\mathrm{CHCHCH}_{3}\right), 110.5($ Het $\mathrm{Ar} \mathrm{CH}), 115.6\left(\mathrm{RCH}=\mathrm{CH}_{2}\right), 119.9($ Het $\mathrm{Ar} \mathrm{CH}), 124.3$ 
(Het $\mathrm{Ar} \mathrm{CH}$ ), 124.6 (Het $\mathrm{Ar} \mathrm{CH}), 141.1\left(\mathrm{RCH}=\mathrm{CH}_{2}\right), 141.2$ (quat. $\left.\mathrm{Ar} \mathrm{C}\right), 150.8$ (quat $\mathrm{Ar}$ C), $170.0(\mathrm{~N}=\mathrm{CO})$; minor diastereomer: $14.8\left(\mathrm{R}_{2} \mathrm{CHCH}_{3}\right), 16.6\left(\mathrm{CH}_{2}=\mathrm{CHCHCH}_{3}\right), 39.6$ $\left(\mathrm{R}_{2} \mathrm{CHCH}_{3}\right), 41.9\left(\mathrm{CH}_{2}=\mathrm{CHCHCH}_{3}\right), 110.5(\mathrm{Het} \mathrm{Ar} \mathrm{CH}), 115.1\left(\mathrm{RCH}=\mathrm{CH}_{2}\right), 119.9(\mathrm{Het}$ $\mathrm{Ar} \mathrm{CH}), 124.2$ (Het $\mathrm{Ar} \mathrm{CH}), 124.6(\mathrm{Het} \mathrm{Ar} \mathrm{CH}), 141.1\left(\mathrm{RCH}=\mathrm{CH}_{2}\right), 141.3$ (quat. Ar C), 141.4 (quat. $\mathrm{Ar} \mathrm{C}), 170.0(\mathrm{~N}=\mathrm{CO})$; IR $\left(\mathrm{CH}_{2} \mathrm{Cl}_{2}\right)$ v $\max$ : 3052, 2983, 1780, 1640, 1612, 1566, 1456, 1421, 1264, 1147, 1069, 1003, 922, 896; HRMS calcd for [M+H] 202.1232, found 202.1226 .

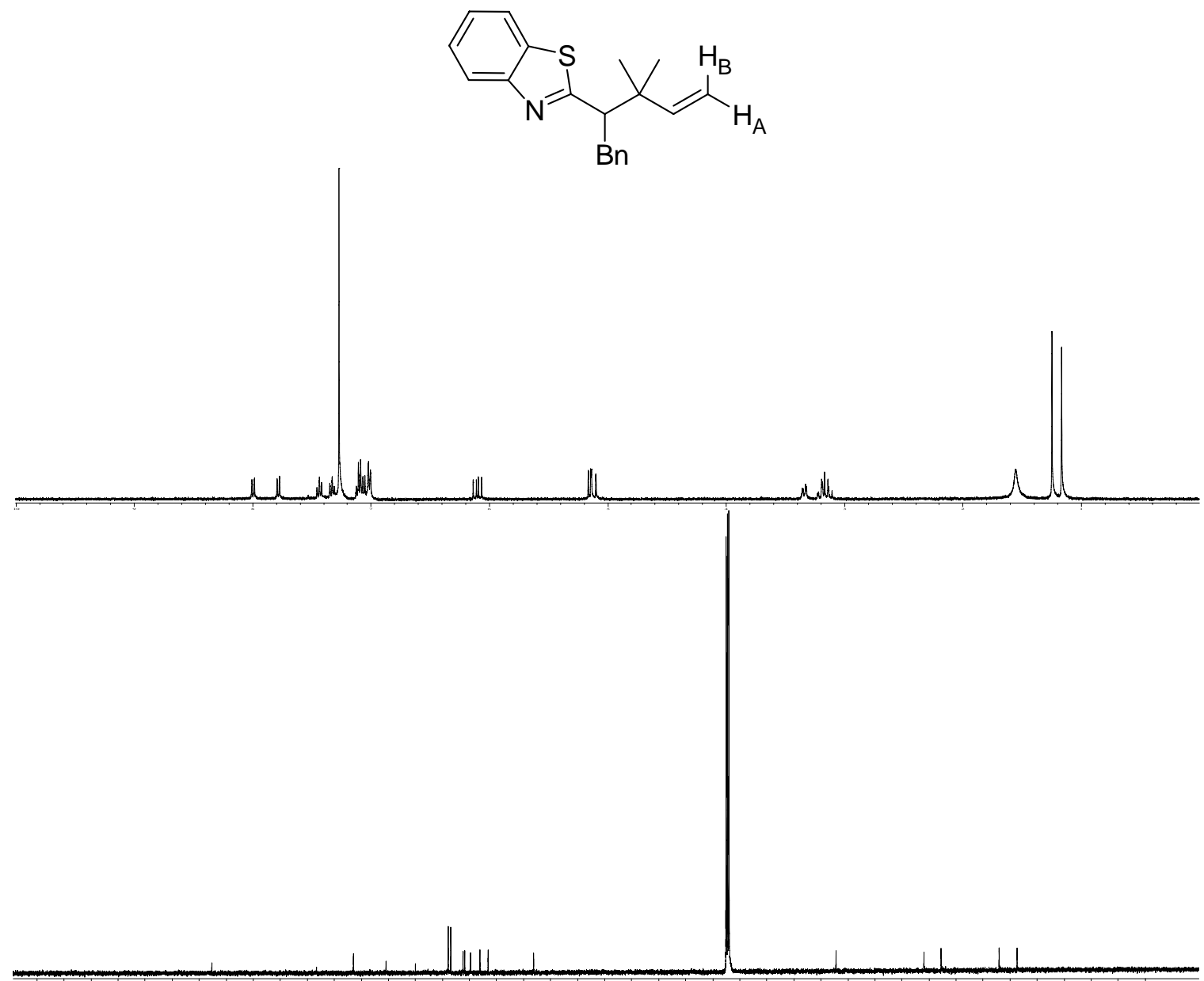

2n: (2-(3,3-dimethyl-1-phenylpent-4-en-2-yl)benzothiazole): colorless oil: $\quad 98: 2$ hex/EtOAc: ${ }^{1} \mathrm{H}$ NMR $\left(400 \mathrm{MHz}, \mathrm{CDCl}_{3}\right) \delta 1.16$ (s, 3H: one of diastereotopic $\left.\mathrm{R}_{2} \mathrm{C}\left(\mathrm{CH}_{3}\right)_{2}\right), 1.25$ (s, 3H: one of diastereotopic $\left.\mathrm{R}_{2} \mathrm{C}\left(\mathrm{CH}_{3}\right)_{2}\right), 3.17$ (m, $\left.2 \mathrm{H}: \mathrm{R}_{2} \mathrm{C}\left(\mathrm{CH}_{2} \mathrm{Ph}\right)_{2}\right)$, 3.34 (dd, $1 \mathrm{H}: J=3.4,11.4 \mathrm{~Hz}, \mathrm{R}_{2} \mathrm{CHBn}$ ), 5.12 (dd, $1 \mathrm{H}: J=1.0,17.3 \mathrm{~Hz}, \mathrm{H}_{\mathrm{B}}$ ), 5.15 (dd, $\left.1 \mathrm{H}: J=1.0,10.7 \mathrm{~Hz}, \mathrm{H}_{\mathrm{A}}\right), 6.10\left(\mathrm{dd}, 1 \mathrm{H}: J=10.7,17.3 \mathrm{~Hz}, \mathrm{RCH}=\mathrm{CH}_{2}\right), 7.01(\mathrm{~m}, 2 \mathrm{H}$ : overlapping Ar CH's), 7.09 (m, 3H: overlapping Ar CH's), 7.33 (app. t, $1 \mathrm{H}: J=8.1 \mathrm{~Hz}$, Het $\mathrm{ArCH}$ ), 7.43 (app. t, $1 \mathrm{H}: J=8.1 \mathrm{~Hz}$, Het $\mathrm{ArCH}$ ), 7.78 (d, $1 \mathrm{H}: J=8.1 \mathrm{~Hz}$, Het Ar $\mathrm{CH}$ ), 7.99 (d, $1 \mathrm{H}: J=8.1 \mathrm{~Hz}$, Het $\mathrm{Ar} \mathrm{CH}) ;{ }^{13} \mathrm{C}$ NMR $\left(125 \mathrm{MHz}, \mathrm{CDCl}_{3}\right) \delta 23.6$ (one of diastereotopic $\left.\mathrm{R}_{2} \mathrm{C}\left(\mathrm{CH}_{3}\right)_{2}\right), 27.0$ (one of diastereotopic $\left.\mathrm{R}_{2} \mathrm{C}\left(\mathrm{CH}_{3}\right)_{2}\right), 37.7\left(\mathrm{RCH}_{2} \mathrm{Ph}\right), 40.9$ $\left(\mathrm{R}_{2} \mathrm{C}\left(\mathrm{CH}_{3}\right)_{2}\right), 57.1\left(\mathrm{R}_{2} \mathrm{CHBn}\right), 113.1\left(\mathrm{RCH}=\mathrm{CH}_{2}\right), 121.5(\mathrm{Het} \mathrm{Ar} \mathrm{CH}), 123.0(\mathrm{Het} \mathrm{Ar}$ $\mathrm{CH}), 124.7(\mathrm{Het} \mathrm{Ar} \mathrm{CH}), 125.8(\mathrm{Het} \mathrm{Ar} \mathrm{CH}), 126.1(\mathrm{Ar} \mathrm{CH}), 128.4(\mathrm{Ar} \mathrm{CH}), 128.9(\mathrm{Ar}$ $\mathrm{CH}$ ), 134.9 (quat. $\mathrm{Ar}$ C), 140.4 (quat. $\mathrm{Ar} \mathrm{C}$ ), $146.4\left(\mathrm{RCH}=\mathrm{CH}_{2}\right), 153.2$ (quat. $\mathrm{Ar} \mathrm{C}$ ), 
$172.6(\mathrm{~N}=\mathrm{CS}) ; \mathrm{IR}\left(\mathrm{CH}_{2} \mathrm{Cl}_{2}\right) v_{\max }: 3054,2986,1603,1550,1501,1437,1421,1265,920$, 896; HRMS calcd for $[\mathrm{M}+\mathrm{H}] 308.1473$, found 308.1477.
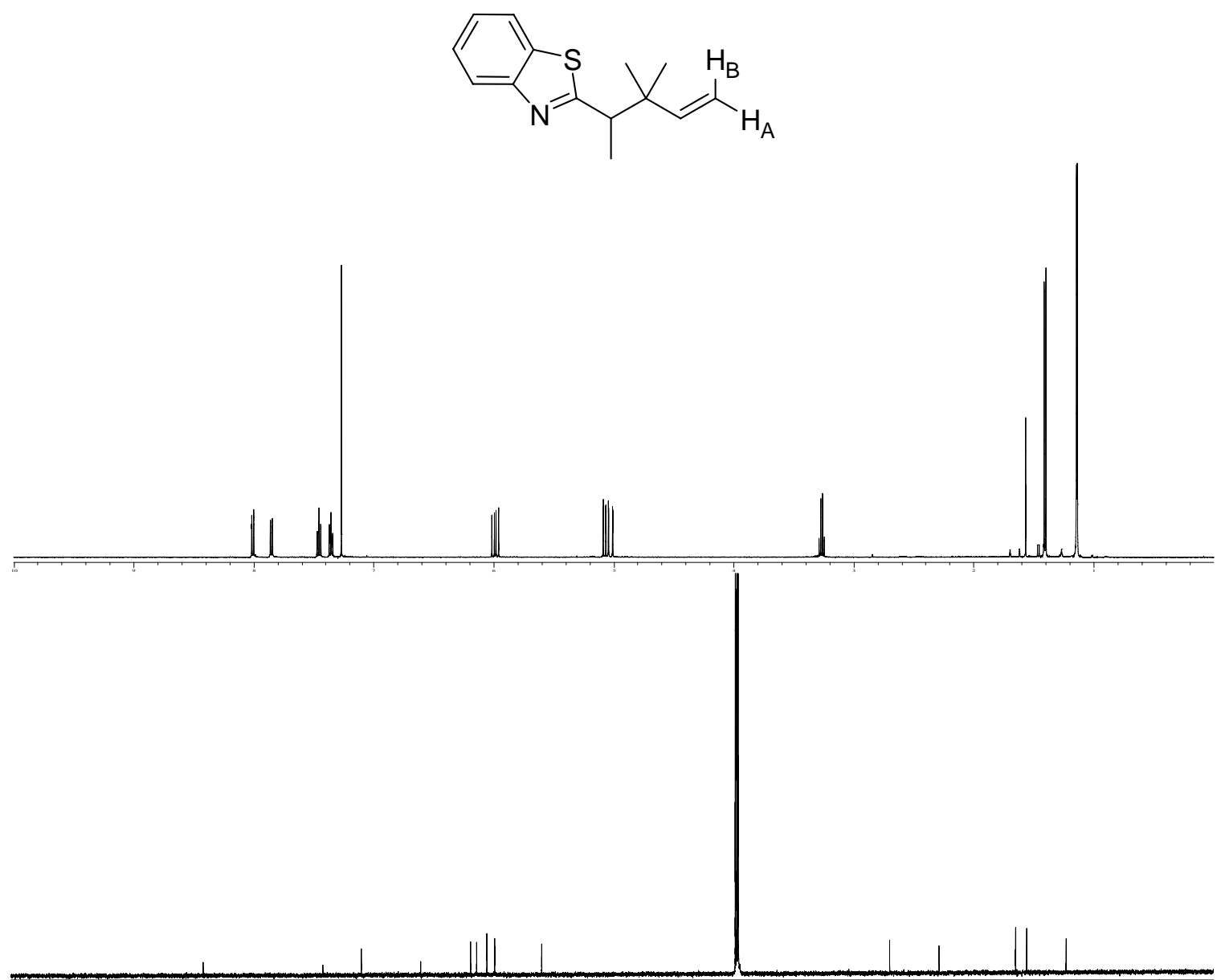

2o: (2-(3,3-dimethylpent-4-en-2-yl)benzothiazole): pale yellow oil: 98:2 hex/EtOAc: ${ }^{1} \mathrm{H}$

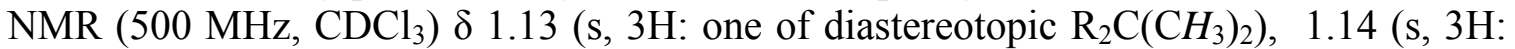
one of diastereotopic $\left.\mathrm{R}_{2} \mathrm{C}\left(\mathrm{CH}_{3}\right)_{2}\right), 1.40$ (d, $3 \mathrm{H}: J=7.1 \mathrm{~Hz}, \mathrm{R}_{2} \mathrm{CHCH}_{3}$ ), 3.27 (q, $1 \mathrm{H}: J=$ $7.1 \mathrm{~Hz}, \mathrm{R}_{2} \mathrm{CHCH}_{3}$ ), $5.03\left(\mathrm{dd}, 1 \mathrm{H}: J=1.3,17.4 \mathrm{~Hz}, \mathrm{H}_{\mathrm{B}}\right.$ ), 5.08 (dd, $1 \mathrm{H}: J=1.3,10.9 \mathrm{~Hz}$, $\mathrm{H}_{\mathrm{A}}$ ), $5.99\left(\mathrm{dd}, 1 \mathrm{H}: J=10.9,17.4 \mathrm{~Hz}, \mathrm{RCH}=\mathrm{CH}_{2}\right), 7.36$ (ddd, $1 \mathrm{H}: J=1.2,7.2,8.1 \mathrm{~Hz}$, Het $\mathrm{Ar} \mathrm{CH}$ ), 7.46 (ddd, $1 \mathrm{H}: J=1.2,7.2,8.1 \mathrm{~Hz}$, Het $\mathrm{Ar} \mathrm{CH}$ ), 7.85 (d, 1H: $J=8.1 \mathrm{~Hz}$, Het Ar $\mathrm{CH}), 8.01(\mathrm{~d}, 1 \mathrm{H}: J=8.1 \mathrm{~Hz}$, Het $\mathrm{Ar} \mathrm{CH}) ;{ }^{13} \mathrm{C}$ NMR $\left(125 \mathrm{MHz}, \mathrm{CDCl}_{3}\right) \delta 17.0$ $\left(\mathrm{R}_{2} \mathrm{CHCH}_{3}\right.$ ), 24.2 (one of diastereotopic $\mathrm{R}_{2} \mathrm{C}\left(\mathrm{CH}_{3}\right)_{2}$ ), 26.2 (one of diastereotopic $\left.\mathrm{R}_{2} \mathrm{C}\left(\mathrm{CH}_{3}\right)_{2}\right), 40.2\left(\mathrm{R}_{2} \mathrm{C}\left(\mathrm{CH}_{3}\right)_{2}\right), 49.2\left(\mathrm{R}_{2} \mathrm{CHCH}_{3}\right), 112.9\left(\mathrm{RCH}=\mathrm{CH}_{2}\right), 121.5($ Het $\mathrm{Ar} \mathrm{CH})$, 122.9 (Het $\mathrm{Ar} \mathrm{CH}$ ), 124.8 (Het $\mathrm{Ar} \mathrm{CH}), 125.9$ (Het $\mathrm{Ar} \mathrm{CH}$ ), 135.1 (quat. Het $\mathrm{Ar} \mathrm{C}$ ), 145.9 $\left(\mathrm{RCH}=\mathrm{CH}_{2}\right), 153.0$ (quat. Het $\mathrm{ArC}$ ), $174.9(\mathrm{~N}=\mathrm{CS}) ; \mathrm{IR}\left(\mathrm{CH}_{2} \mathrm{Cl}_{2}\right)$ v max $: 3054,2979,1637$, 1506, 1455, 1437, 1421, 1265, 1048, 1004, 918, 896; HRMS calcd for [M+H] 232.1160, found 232.1158 . 

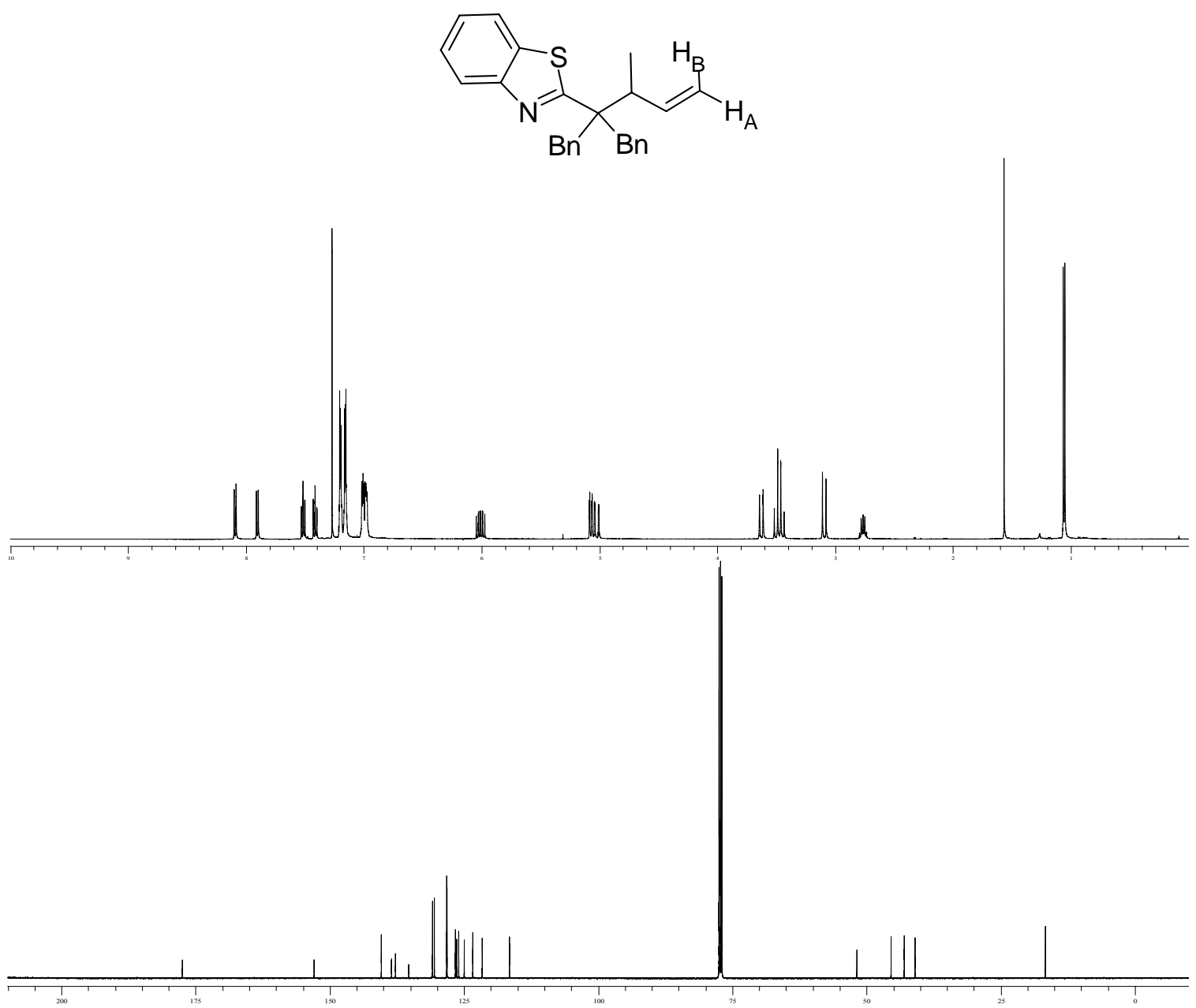

2p: (2-(2-benzyl-3-methyl-1-phenylpent-4-en-2-yl)benzothiazole): pale yellow oil: 99.5:0.5 hex/EtOAc: ${ }^{1} \mathrm{H}$ NMR (500 MHz, $\left.\mathrm{CDCl}_{3}\right) \delta 1.06$ (d, 3H: $J=6.8 \mathrm{~Hz}, \mathrm{R}_{2} \mathrm{CHCH}_{3}$ ), $2.76\left(\mathrm{dq}, 1 \mathrm{H}: J=6.8,8.7 \mathrm{~Hz}, \mathrm{R}_{2} \mathrm{CCHCH}_{3}\right), 3.09(\mathrm{~d}, 1 \mathrm{H}: J=15.4 \mathrm{~Hz}$, one of diastereotopic $\mathrm{R}_{2} \mathrm{C}(\mathrm{CHHPh})_{2}$ ), 3.47 (app. q, $2 \mathrm{H}: J=14.4 \mathrm{~Hz}$, diastereotopic $\left.\mathrm{R}_{2} \mathrm{C}(\mathrm{CHHPh})_{2}\right), 3.63\left(\mathrm{~d}, 1 \mathrm{H}: J=15.4 \mathrm{~Hz}\right.$, one of diastereotopic $\left.\mathrm{R}_{2} \mathrm{C}(\mathrm{CHHPh})_{2}\right), 5.02$ (dd, $1 \mathrm{H}: J=1.7,17.1 \mathrm{~Hz}, \mathrm{H}_{\mathrm{B}}$ ), 5.07 (dd, $1 \mathrm{H}: J=1.7,10.2 \mathrm{~Hz}, \mathrm{H}_{\mathrm{A}}$ ), 6.01 (ddd, $1 \mathrm{H}: J=$ 8.8, 10.2, 17.1 Hz, $\left.\mathrm{RCH}=\mathrm{CH}_{2}\right), 6.98(\mathrm{~m}, 2 \mathrm{H}$ : overlapping $\mathrm{Ar} \mathrm{CH}$ 's), $7.01(\mathrm{~m}, 2 \mathrm{H}$ : overlapping Ar CH's), 7.16 (m, 3H: overlapping Ar CH's), 7.20 (m, 3H: Ar CH's), 7.41 (ddd, $1 \mathrm{H}: J=0.9,7.4,8.1 \mathrm{~Hz}$, Het Ar CH), 7.52 (ddd, $1 \mathrm{H}: J=1.2,7.4,8.1 \mathrm{~Hz}$, Het $\mathrm{Ar}$ $\mathrm{CH}), 7.90(\mathrm{~d}, 1 \mathrm{H}: J=8.1 \mathrm{~Hz}$, Het Ar CH), 8.09 (d, 1H: $J=8.1 \mathrm{~Hz}$, Het $\mathrm{ArCH}) ;{ }^{13} \mathrm{C}$ NMR $\left(125 \mathrm{MHz}, \mathrm{CDCl}_{3}\right) \delta 16.8\left(\mathrm{R}_{2} \mathrm{CHCH}_{3}\right), 41.2$ (diasterotopic $\left.\mathrm{CH}_{2} \mathrm{Ph}\right), 43.2$ (diastereotopic $\left.\mathrm{CH}_{2} \mathrm{Ph}\right), 45.7\left(\mathrm{R}_{2} \mathrm{CHCH}_{3}\right), 52.1\left(\mathrm{R}_{2} \mathrm{C}\left(\mathrm{CH}_{2} \mathrm{Ph}\right)_{2}\right), 117.2\left(\mathrm{RCH}=\mathrm{CH}_{2}\right), 122.3$ (Het $\mathrm{Ar} \mathrm{CH}), 124.1(\mathrm{Het} \mathrm{Ar} \mathrm{CH}), 125.7(\mathrm{Het} \mathrm{Ar} \mathrm{CH}), 126.7(\mathrm{Het} \mathrm{ArCH}), 127.1(\mathrm{Ar} \mathrm{CH})$, 127.4 (Ar CH), 128.9 ( $\mathrm{Ar} \mathrm{CH}), 129.0(\mathrm{Ar} \mathrm{CH}), 131.3(\mathrm{Ar} \mathrm{CH}), 131.7(\mathrm{Ar} \mathrm{CH}), 136.1$ (quat Ar. C), 138.6 (quat Ar. C), 139.3 (quat. $\mathrm{Ar} \mathrm{C}$ ), $141.3\left(\mathrm{RCH}=\mathrm{CH}_{2}\right), 153.8$ (quat. $\mathrm{Ar}$ C), $178.5(\mathrm{~N}=\mathrm{CS})$; IR $\left(\mathrm{CH}_{2} \mathrm{Cl}_{2}\right)$ v $\max : 3055,2985,1635,1603,1550,1496,1453,1437$, 1421, 1263, 1004, 951, 919, 896; HRMS calcd for [M+H] 384.1786, found 384.1785. 


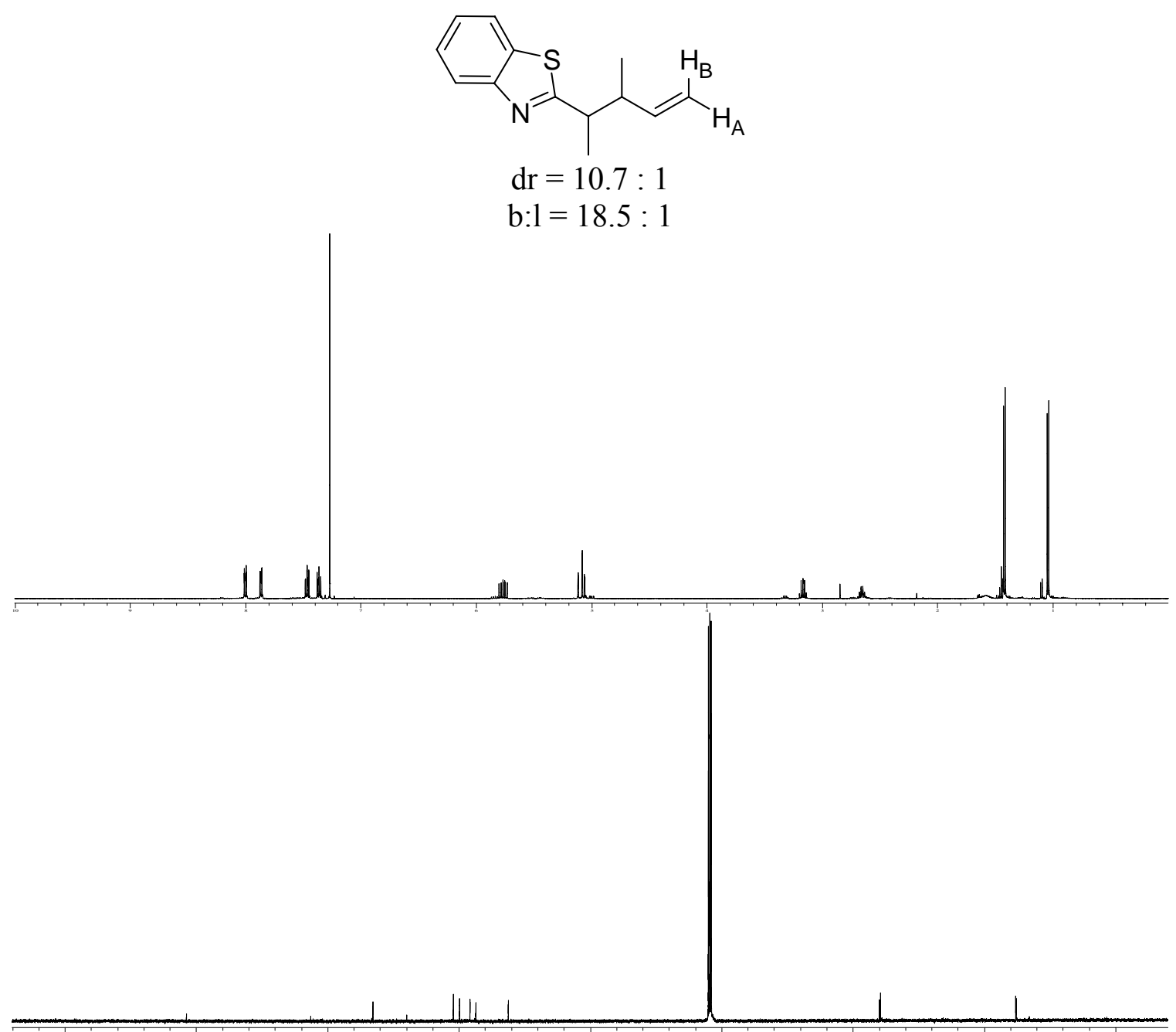

2q: (2-(3-methypent-4-en-2-yl)benzothiazole): yellow oil: 98:2 hex/EtOAc: ${ }^{1} \mathrm{H}$ NMR $\left(500 \mathrm{MHz}, \mathrm{CDCl}_{3}\right) \delta$ major diastereomer: $1.04\left(\mathrm{~d}, 3 \mathrm{H}: J=6.9 \mathrm{~Hz}, \mathrm{CH}_{2}=\mathrm{CHCHCH}_{3}\right)$, 1.42 (d, 3H: $J=7.0 \mathrm{~Hz}, \mathrm{R}_{2} \mathrm{CHCH}_{3}$ ), 2.65 (sext., $1 \mathrm{H}: J=6.9 \mathrm{~Hz}, \mathrm{CH}_{2}=\mathrm{CHCHCH}_{3}$ ), 3.17 (dq, 1H: $\left.J=6.9,7.8 \mathrm{~Hz}, \mathrm{R}_{2} \mathrm{CHCH}_{3}\right), 5.06\left(\mathrm{dd}, 1 \mathrm{H}: J=1.6,10.3 \mathrm{~Hz}, \mathrm{H}_{\mathrm{A}}\right), 5.09$ (dd, $1 \mathrm{H}: J$ $\left.=1.6,17.2 \mathrm{~Hz}, \mathrm{H}_{\mathrm{B}}\right), 5.77\left(\mathrm{ddd}, 1 \mathrm{H}: J=8.3,10.3,17.2 \mathrm{~Hz}, \mathrm{RCH}=\mathrm{CH}_{2}\right), 7.36$ (ddd, $1 \mathrm{H}: J$ $=1.3,7.3,8.2 \mathrm{~Hz}$, Het Ar CH), 7.46 (ddd, 1H: $J=1.3,7.3,8.2 \mathrm{~Hz}$, Het Ar CH), 7.86 (app. d, 1H: $J=8.2 \mathrm{~Hz}$, Het Ar CH), 8.00 (app. d, 1H: $J=8.2 \mathrm{~Hz}$, Het Ar CH); minor diastereomer: 1.10 (d, 3H: $\left.J=7.1 \mathrm{~Hz}, \mathrm{CH}_{2}=\mathrm{CHCHCH}_{3}\right), 1.44(\mathrm{~d}, 3 \mathrm{H}: J=7.1 \mathrm{~Hz}$, $\mathrm{R}_{2} \mathrm{CHCH}_{3}$ ), 2.73 (sext., $1 \mathrm{H}: J=7.1 \mathrm{~Hz}, \mathrm{CH}_{2}=\mathrm{CHCHCH}_{3}$ ), 3.32 (app. p, $1 \mathrm{H}: J=7.1 \mathrm{~Hz}$, $\mathrm{R}_{2} \mathrm{CHCH}_{3}$ ), 4.99 (dt, 1H: $\left.J=1.4,10.2 \mathrm{~Hz}, \mathrm{H}_{\mathrm{B}}\right), 5.03$ (dt, $\left.1 \mathrm{H}: 1.4,17.3 \mathrm{~Hz}, \mathrm{H}_{\mathrm{B}}\right), 5.83$ (ddd, $1 \mathrm{H}: J=7.3,10.2,17.3 \mathrm{~Hz}, \mathrm{RCH}=\mathrm{CH}_{2}$ ), 7.36 (ddd, $1 \mathrm{H}: J=1.3,7.3,8.2 \mathrm{~Hz}$, Het Ar $\mathrm{CH}$ ), 7.46 (ddd, 1H: $J=1.3,7.3,8.2 \mathrm{~Hz}$, Het Ar CH), 7.86 (app. d, 1H: $J=8.2 \mathrm{~Hz}$, Het Ar CH), 8.00 (app. d, $1 \mathrm{H}: J=8.2 \mathrm{~Hz}$, Het $\mathrm{Ar} \mathrm{CH}$ ); ${ }^{13} \mathrm{C}$ NMR (125 MHz, $\left.\mathrm{CDCl}_{3}\right) \delta$ major diastereomer: $18.7 \quad\left(\mathrm{R}_{2} \mathrm{CHCH}_{3}\right), 18.8 \quad\left(\mathrm{CH}_{2}=\mathrm{CHCHCH}_{3}\right), \quad 44.5 \quad\left(\mathrm{R}_{2} \mathrm{CHCH}_{3}\right), 44.7$ $\left(\mathrm{CH}_{2}=\mathrm{CHCHCH}_{3}\right), 115.4\left(\mathrm{RCH}=\mathrm{CH}_{2}\right), 121.5($ Het Ar CH$), 122.7($ Het Ar CH$), 124.6$ (Het $\mathrm{Ar} \mathrm{CH}$ ), 125.8 (Het $\mathrm{Ar} \mathrm{CH}), 134.7$ (quat. Het $\mathrm{Ar} \mathrm{C}$ ), $141.1\left(\mathrm{RCH}=\mathrm{CH}_{2}\right), 153.0$ (quat. Het $\mathrm{Ar} \mathrm{C}), 176.6(\mathrm{~N}=\mathrm{CS})$; IR $\left(\mathrm{CH}_{2} \mathrm{Cl}_{2}\right)$ v $\max$ : 3053, 2983, 2930, 1695, 1641, 1514, 1455, 1438, 1421, 1268, 1042, 999, 920, 896; HRMS calcd for [M+H] 218.1003, found 218.0993. 


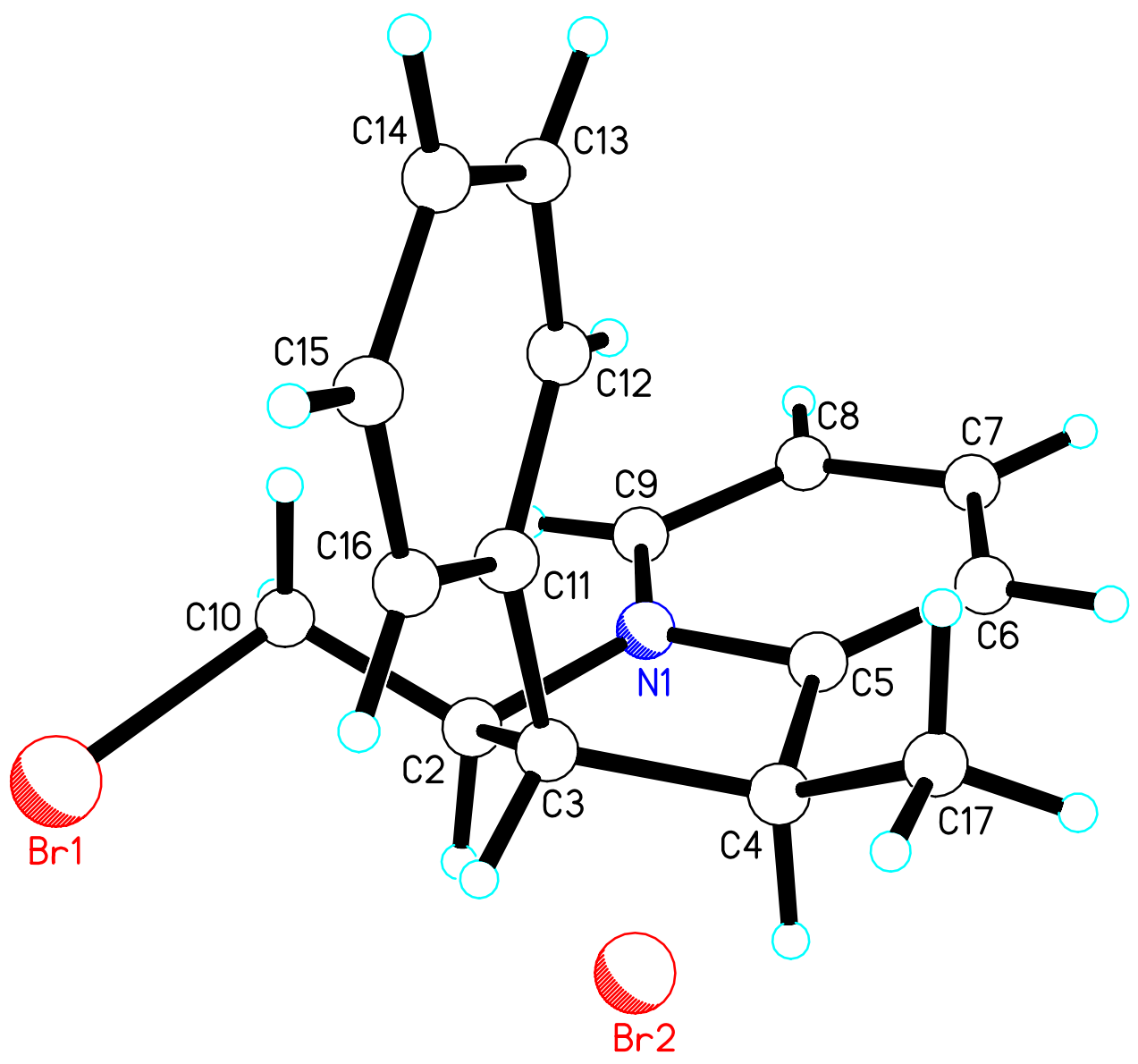

Crystal Structure for $\mathbf{4 g}$ 